\title{
THE NONCOMMUTATIVE GEOMETRY OF THE LANDAU HAMILTONIAN: DIFFERENTIAL ASPECTS
}

\author{
GIUSEPPE DE NITTIS AND MAXIMILIANO SANDOVAL
}

\begin{abstract}
In this work we study the differential aspects of the noncommutative geometry for the magnetic $\mathrm{C}^{*}$-algebra which is a 2-cocycle deformation of the group $\mathrm{C}^{*}$-algebra of $\mathbb{R}^{2}$. This algebra is intimately related to the study of the Quantum Hall Effect in the continuous, and our results aim to provide a new geometric interpretation of the related Kubo's formula. Taking inspiration from the ideas developed by Bellissard during the 80 's, we build an appropriate Fredholm module for the magnetic $\mathrm{C}^{*}$-algebra based on the magnetic Dirac operator which is the square root (à la Dirac) of the quantum harmonic oscillator. Our main result consist of establishing an important piece of Bellissard's theory, the so-called second Connes' formula. In order to do so, we establish the equality of three cyclic 2-cocycles defined on a dense subalgebra of the magnetic $\mathrm{C}^{*}$-algebra. Two of these 2-cocycles are new in the literature and are defined by Connes' quantized differential calculus, with the use of the Dixmier trace and the magnetic Dirac operator.
\end{abstract}

MSC 2010: Primary: 81Q10; Secondary: 81Q05, 81Q15, 33C1.

Keywords: Landau Hamiltonian, spectral triple, Dixmier trace, Connes' formulas.

\section{CONTENTS}

1. Introduction 2

1.1. Background material and known results 2

1.2. New results 6

2. Quantized calculus of the magnetic spectral triple 13

2.1. Quasi-even Fredholm module 13

2.2. Quasi-differential structure 15

$\begin{array}{ll}\text { 2.3. Quasi-cycles } & 17\end{array}$

2.4. Chern character 19

3. Direct proof of the Second Connes' formula 20

Appendix A. Technicalities 25

A.1. Weak $L^{p}$-spaces 25

A.2. Relevant trace-class elements 27

A.3. Quasi-symmetry of the Dirac operator and its consequences 30

Appendix B. The cyclic cohomology of the magnetic algebra 31

References 33

Date: December 17, 2021. 


\section{INTRODUCTION}

This work continues the study of the noncommutative geometry of the magnetic $\mathrm{C}^{*}$ algebra $\mathscr{C}_{\mathrm{B}}$ associated with the Landau Hamiltonian started in [DS]. While the previous work has been devoted to the analysis of metric aspects, in the present work we will investigate the topological properties by developing an appropriate quantized calculus based on the spectral triple introduced in [DS] and endowed with a suitable grading. The main result of this paper is the proof of the equality of three cyclic 2-cocycles $\Psi_{\mathrm{B}}, \mathfrak{C h}_{\mathrm{B}}$ and $\tau_{\mathrm{B}, 2}$ defined on a dense subalgebra of $\mathscr{C}_{\mathrm{B}}$. The 2-cocycle $\Psi_{\mathrm{B}}$ is standard in the literature concerning the topology of $\mathscr{C}_{\mathrm{B}}$ while $\mathfrak{C h}_{\mathrm{B}}$ and $\tau_{\mathrm{B}, 2}$ are new and are defined by Connes' quantized differential calculus, with the use of the Dixmier trace and the the spectral triple introduced in [DS]. The equalities $\Psi_{\mathrm{B}}=\mathfrak{C h}_{\mathrm{B}}$ and $\Psi_{\mathrm{B}}=\tau_{\mathrm{B}, 2}$, called the second Connes' formulae in agreement with the name used in the seminal paper [BES], provide a new way of representing the Kubo's formula for the Quantum Hall effect inside the noncommutative geometry of the magnetic $C^{*}$-algebra $\mathscr{C}_{\mathrm{B}}$. In particular, the construction of $\tau_{\mathrm{B}, 2}$ requires the introduction of the notion of quasi-even Fredholm which can be considered as a new idea in noncommutative geometry extending the usual concept of Fredholm module. Our hope is that this idea could be of some interest also for further applications in noncommutative geometry. In the rest of this introduction we will give a more detailed account of our results by comparing them with the existing literature.

1.1. Background material and known results. In order to describe the main results of this work, we will first proceed to introduce the necessary background. The material and the notation presented below are borrowed from [DGM, DS].

Consider the Hilbert space $\mathrm{L}^{2}\left(\mathbb{R}^{2}\right)$, and let $\left\{\psi_{n, m}\right\} \subset \mathrm{L}^{2}\left(\mathbb{R}^{2}\right)$, with $n, m \in \mathbb{N}_{0}:=$ $\mathbb{N} \cup\{0\}$, be the orthonormal basis provided by the generalized Laguerre basis defined by

$$
\psi_{n, m}(x):=\psi_{0,0}(x) \sqrt{\frac{n !}{m !}}\left[\frac{x_{1}+i x_{2}}{\sqrt{2} \ell_{B}}\right]^{m-n} L_{n}^{(m-n)}\left(\frac{|x|^{2}}{2 \ell_{B}^{2}}\right),
$$

where

$$
\mathrm{L}_{n}^{(\alpha)}(\zeta):=\sum_{j=0}^{n} \frac{(\alpha+n)(\alpha+n-1) \ldots(\alpha+j+1)}{j !(n-j) !}(-\zeta)^{j}, \quad \alpha, \zeta \in \mathbb{R}
$$

are the generalized Laguerre polynomial of degree $m$ (with the usual convention $0 !=1$ ) and

$$
\psi_{0,0}(x):=\frac{1}{\sqrt{2 \pi \ell_{B}}} \mathrm{e}^{-\frac{|x|^{2}}{4 \ell_{\mathrm{B}}^{2}}} .
$$

The parameter $\ell_{B}>0$ is called magnetic length and the (singular) limit $\ell_{B} \rightarrow+\infty$ corresponds to the limit where the magnetic field $B$ vanishes. Let us introduce the family $\left\{\Upsilon_{j \mapsto k} \mid(j, k) \in \mathbb{N}_{0}^{2}\right\}$ of transition operators on $L^{2}\left(\mathbb{R}^{2}\right)$ defined by

$$
\Upsilon_{j \mapsto k} \psi_{n, m}:=\delta_{j, n} \psi_{k, m}, \quad k, j, n, m \in \mathbb{N}_{0} \text {. }
$$


A direct computation [DS, Proposition 2.10] provides

$$
\left(\Upsilon_{j \mapsto k}\right)^{*}=\Upsilon_{k \mapsto j}, \quad \Upsilon_{j \mapsto k} \Upsilon_{m \mapsto n}=\delta_{j, n} \Upsilon_{m \mapsto k},
$$

and with these rules in hand one can define the magnetic $\mathrm{C}^{*}$-algebra

$$
\mathscr{C}_{\mathrm{B}}=\mathrm{C}^{*}\left(\Upsilon_{\mathrm{j} \mapsto k}, k, j \in \mathbb{N}_{0}\right)
$$

as the $\mathrm{C}^{*}$-algebra generated by the transition operators. This name is justified by the fact that the Landau projections

$$
\Pi_{j}:=\Upsilon_{k \mapsto j} \Upsilon_{j \mapsto k}=\sum_{r \in \mathbb{N}_{0}}\left|\psi_{j, r}\right\rangle\left\langle\psi_{j, r}\right|, \quad j \in \mathbb{N}_{0}
$$

(independently of k) are elements of $\mathscr{C}_{\mathrm{B}}$, the latter being the spectral projection of the Landau Hamiltonian

$$
\mathrm{H}_{\mathrm{B}}=\frac{\epsilon_{\mathrm{B}}}{2}\left(\mathrm{~K}_{1}^{2}+\mathrm{K}_{2}^{2}\right)
$$

where

$$
\mathrm{K}_{1}=-\mathrm{i} \ell_{\mathrm{B}} \frac{\partial}{\partial x_{1}}-\frac{1}{2 \ell_{\mathrm{B}}} \mathrm{x}_{2}, \quad \mathrm{~K}_{2}=-\mathrm{i} \ell_{\mathrm{B}} \frac{\partial}{\partial x_{2}}+\frac{1}{2 \ell_{\mathrm{B}}} \mathrm{x}_{1}
$$

are the magnetic momenta and the constant $\epsilon_{\mathrm{B}}$ is the fundamental magnetic energy.

There are interesting spaces of operators contained in $\mathscr{C}_{\mathrm{B}}$. Let us introduce the following notation

$$
\begin{aligned}
& \mathscr{S}_{\mathrm{B}}:=\left\{A:=\sum_{(j, k) \in \mathbb{N}_{0}^{2}} a_{j, k} \Upsilon_{j \mapsto k} \mid\left\{a_{j, k}\right\} \in S\left(\mathbb{N}_{0}^{2}\right)\right\}, \\
& \mathscr{L}_{\mathrm{B}}^{\mathrm{p}}:=\left\{A:=\sum_{(j, k) \in \mathbb{N}_{0}^{2}} a_{j, k} \Upsilon_{j \mapsto k} \mid\left\{a_{j, k}\right\} \in \ell^{p}\left(\mathbb{N}_{0}^{2}\right)\right\},
\end{aligned}
$$

where $S\left(\mathbb{N}_{0}^{2}\right)$ is the space of rapidly decreasing sequences, and $\ell^{p}\left(\mathbb{N}_{0}^{2}\right)$ are the usual discrete $\mathrm{L}^{p}$ spaces. It turns out that [DS, Proposition 2.17]

$$
\mathscr{S}_{\mathrm{B}} \subset \mathscr{L}_{\mathrm{B}}^{1} \subset \mathscr{I}_{\mathrm{B}} \subset \mathscr{L}_{\mathrm{B}}^{2} \subset \mathscr{C}_{\mathrm{B}} \subset \mathscr{M}_{\mathrm{B}}
$$

where

$$
\mathscr{I}_{\mathrm{B}}:=\left\{\mathrm{S}=\mathrm{AB} \mid \mathrm{A}, \mathrm{B} \in \mathscr{L}_{\mathrm{B}}^{2}\right\} \equiv\left(\mathscr{L}_{\mathrm{B}}^{2}\right)^{2}
$$

and $\mathscr{M}_{\mathrm{B}}$ is the enveloping von Neumann algebra of $\mathscr{C}_{\mathrm{B}}$. All these subspaces are dense in $\mathscr{C}_{\mathrm{B}}$ with respect to the operator norm, and in $\mathscr{M}_{\mathrm{B}}$ with respect to the weak or strong operator topologies. Both $\mathscr{L}_{\mathrm{B}}^{2}$, and consequently $\mathscr{I}_{\mathrm{B}}$, are self-adjoint two-sided ideals of $\mathscr{M}_{\mathrm{B}}$. The spaces $\mathscr{S}_{\mathrm{B}}$ and $\mathscr{L}_{\mathrm{B}}^{2}$ admit special characterizations in terms of integral kernel operators. Let us start with $\mathscr{L}_{\mathrm{B}}^{2}$ (cf. [DS, Section 2.4]). One gets that $\mathrm{A} \in \mathscr{L}_{\mathrm{B}}^{2}$, if and only if, there is a function $f_{A} \in L^{2}\left(\mathbb{R}^{2}\right)$ such that

$$
(A \varphi)(x)=\frac{1}{2 \pi \ell_{B}^{2}} \int_{\mathbb{R}^{2}} \mathrm{~d} y f_{A}(y-x) \Phi_{B}(x, y) \varphi(y), \quad \forall \varphi \in L^{2}\left(\mathbb{R}^{2}\right)
$$


where the function

$$
\Phi_{\mathrm{B}}(x, y):=\mathrm{e}^{\mathrm{i} \frac{x_{1} y_{2}-x_{2} y_{1}}{2 \ell_{\mathrm{B}}^{2}}}, \quad x, y \in \mathbb{R}^{2}
$$

is known as magnetic 2-cocycle. The relation between the integral kernel $f_{A}$ and the sequence $\left\{a_{j, k}\right\} \in \ell^{2}\left(\mathbb{N}_{0}^{2}\right)$ which identifies the expansion of $A$ in the basis $\Upsilon_{j \mapsto k}$ is given by

$$
f_{A}=\sqrt{2 \pi} \ell_{B} \sum_{(j, k) \in \mathbb{N}_{0}^{2}}(-1)^{j-k} a_{j, k} \psi_{k, j}
$$

and the norm bound $\sqrt{2 \pi} l_{\mathrm{B}}\|\mathrm{A}\| \leqslant\left\|\mathrm{f}_{\mathrm{A}}\right\|_{\mathrm{L}^{2}}$ holds true. A similar result holds for $\mathscr{S}_{\mathrm{B}}$, namely $A \in \mathscr{S}_{B}$, if and only if, there is a Schwarz function $f_{A} \in S\left(\mathbb{R}^{2}\right)$ such that $A$ has an integral representation of the type (1.9) and the relation between $A$ and its kernel is given again by (1.10). In addition, $\mathscr{S}_{\mathrm{B}}$ has the structure of a Fréchet pre- $\mathrm{C}^{*}$-algebra of $\mathscr{C}_{\mathrm{B}}$ [DS, Proposition $2.8 \&$ Proposition 2.14]. Behind the integral representation (1.9) there is the fact that $\mathscr{C}_{\mathrm{B}}$ is nothing more than the group $\mathrm{C}^{*}$-algebra of $\mathbb{R}^{2}$ twisted by the cocycle $\Phi_{\mathrm{B}}$ (cf. [DS, Section 2.2] and references therein).

As discussed in [DS, Section 2.6], one can endow the von Neumann algebra $\mathscr{M}_{\mathrm{B}}$ with a remarkable normal, faithful and semi-finite (NFS) trace $f_{\mathrm{B}}$ defined on the ideal $\mathscr{I}_{\mathrm{B}}$, which is uniquely specified by the prescription

$$
f_{\mathrm{B}}\left(\mathrm{A}^{*} \mathrm{~B}\right):=\frac{1}{2 \pi \ell_{\mathrm{B}}^{2}}\left\langle\mathrm{f}_{\mathrm{A}}, \mathrm{f}_{\mathrm{B}}\right\rangle_{\mathrm{L}^{2}}, \quad \forall \mathrm{A}, \mathrm{B} \in \mathscr{L}_{\mathrm{B}}^{2}
$$

where $\langle,\rangle_{L^{2}}$ is the usual inner product in $L^{2}\left(\mathbb{R}^{2}\right)$ and $f_{A}, f_{B} \in L^{2}\left(\mathbb{R}^{2}\right)$ are the integral kernels of $A$ and $B$ respectively, as given by the prescription (1.10). The computation of the trace $f_{\mathrm{B}}$ on elements of the domain $\mathscr{I}_{\mathrm{B}}$ is facilitated by observing that every $S \in \mathscr{I}_{\mathrm{B}}$ has an integral kernel of type (1.10) which satisfies $f_{S} \in L^{2}\left(\mathbb{R}^{2}\right) \cap C_{0}\left(\mathbb{R}^{2}\right)$, where $C_{0}\left(\mathbb{R}^{2}\right)$ is the space of continuous functions which vanish at infinity. On these elements the trace can be computed as $f_{\mathrm{B}}(\mathrm{S})=\mathrm{f}_{\mathrm{S}}(0)$ [DS, Corollary 2.22]. The trace $f_{\mathrm{B}}$ has the physical meaning of a thermodynamic limit. Indeed, one can prove that [DS, Lemma 2.23]

$$
f_{B}(S)=2 \pi \ell_{B}^{2} \lim _{n \rightarrow+\infty} \frac{1}{\left|\Lambda_{n}\right|} \operatorname{Tr}_{L^{2}\left(\mathbb{R}^{2}\right)}\left(\chi_{\wedge_{n}} S \chi_{\Lambda_{n}}\right), \quad S \in \mathscr{I}_{B}
$$

where the family $\left\{\Lambda_{n}\right\}$ provides an increasing sequence of compact subsets $\Lambda_{n} \subseteq \mathbb{R}^{2}$ such that $\Lambda_{n} \nearrow \mathbb{R}^{2}$ and which satisfies the Folner condition (see e.g. [Gree] for more details), $\left|\Lambda_{n}\right|$ is the Lebesgue measure of $\Lambda_{n}$ and $\chi_{\Lambda_{n}}$ is the projection defined as the multiplication operator by the characteristic function of $\Lambda_{n}$. The expression on the righthand side of (1.12) is known as trace per unit of volume.

The magnetic algebra $\mathscr{C}_{\mathrm{B}}$ admits a pair of unbounded spatial derivations which can be initially defined on the pre- $\mathrm{C}^{*}$-algebra $\mathscr{S}_{\mathrm{B}}$ by the commutators

$$
\nabla_{j} A:=-i\left[x_{j}, A\right], \quad j=1,2, \quad A \in \mathscr{S}_{B},
$$


where $x_{j}$ are the position operators on $L^{2}\left(\mathbb{R}^{2}\right)$. By closing with respect to suitable Fréchettype norms one can define the Banach spaces $\mathrm{C}^{\mathrm{N}}\left(\mathscr{C}_{\mathrm{B}}\right)$ of $\mathrm{N}$-times differentiable elements (cf. [DS, Section 2.8]). Remarkably, one has that $\mathscr{S}_{\mathrm{B}} \subset \mathrm{C}^{\infty}\left(\mathscr{C}_{\mathrm{B}}\right)$ is made by smooth elements, namely by elements which can be derived an indefinite number of times.

The K-theory of $\mathscr{C}_{\mathrm{B}}$ is quite simple to compute. From [DS, Proposition 2.11] we know that there is an isomorphism of $\mathrm{C}^{*}$-algebras $\mathscr{C}_{\mathrm{B}} \simeq \mathscr{K}$ where $\mathscr{K}$ is the $\mathrm{C}^{*}$-algebra of compact operators. Since the K-theory is invariant under $\mathrm{C}^{*}$-isomorphisms one immediately gets $\mathrm{K}_{0}\left(\mathscr{C}_{\mathrm{B}}\right) \simeq \mathbb{Z}$ and $\mathrm{K}_{1}\left(\mathscr{C}_{\mathrm{B}}\right)=0$. A more precise description of the $\mathrm{K}_{0}$-group is given by.

$$
\mathrm{K}_{0}\left(\mathscr{C}_{\mathrm{B}}\right) \simeq \mathrm{K}_{0}\left(\mathscr{S}_{\mathrm{B}}\right)=\mathbb{Z}\left[\Pi_{0}\right]
$$

The first isomorphism is justified by the fact that $\mathscr{S}_{\mathrm{B}}$ is a pre-C ${ }^{*}$-algebra of $\mathscr{C}_{\mathrm{B}}$ [GVF, Theorem 3.44] and the last equality follows by an inspection of the isomorphism $\mathscr{C}_{\mathrm{B}} \simeq$ $\mathscr{K}$. It is worth noting that since $\Pi_{0} \in \mathscr{S}_{\mathrm{B}}$ then the $\mathrm{K}$-theory of $\mathscr{C}_{\mathrm{B}}$ is realized inside $\mathscr{S}_{\mathrm{B}}$. Moreover, since all the Landau projections are equivalent (in the sense of von Neumann) $\left[\right.$ BES, Lemma 5] one has that $\left[\Pi_{0}\right]=\left[\Pi_{j}\right]$ for every $j \in \mathbb{N}_{0}$.

The trace $f_{\mathrm{B}}$ is a cyclic 0 -cocycle of the algebra $\mathscr{S}_{\mathrm{B}}$ and so it defines a class $\left[f_{\mathrm{B}}\right] \in$ $\operatorname{HC} C^{\text {even }}\left(\mathscr{S}_{\mathrm{B}}\right)$ in the even cyclic cohomology of $\mathscr{S}_{\mathrm{B}}$ (see Appendix B). Given the canonical pairing $\langle\rangle:, \mathrm{HC}^{\text {even }}\left(\mathscr{S}_{\mathrm{B}}\right) \times \mathrm{K}_{\mathrm{O}}\left(\mathscr{S}_{\mathrm{B}}\right) \rightarrow \mathbb{C}$ between the even cyclic cohomology and the even $\mathrm{K}$-theory one can define the map

$$
\mathrm{gl}_{\mathrm{B}}([\mathrm{P}]):=\left\langle\left[f_{\mathrm{B}}\right],[\mathrm{P}]\right\rangle=f_{\mathrm{B}}(\mathrm{P}), \quad[\mathrm{P}] \in \mathrm{K}_{0}\left(\mathscr{S}_{\mathrm{B}}\right)
$$

where $\mathrm{P} \in \mathscr{S}_{\mathrm{B}}$ is any representative of the class $[\mathrm{P}]$ in view of the fact that the $\mathrm{K}$-theory is entirely realized inside the algebra. The map $\mathrm{gl}_{\mathrm{B}}$ is known as the gap labeling function [Bel1, Bel2], and in our specific case, it provides the group isomorphism

$$
\mathrm{gl}_{\mathrm{B}}: \mathrm{K}_{\mathrm{O}}\left(\mathscr{S}_{\mathrm{B}}\right) \stackrel{\simeq}{\longrightarrow} \mathbb{Z}
$$

generated by $f_{B}\left(\Pi_{0}\right)=1$ [DS, eq. (2.22)]. It is worth mentioning that the last result is a special case of [Xia, Theorem 2.2] when the hull of the potentials collapses to a singleton due to the circumstance that we are considering no electrostatic interactions.

By combining the trace $f_{\mathrm{B}}$ and the derivations $\nabla_{j}$ one gets the cyclic 2-cocycle $\Psi_{\mathrm{B}}$ defined by

$$
\Psi_{\mathrm{B}}\left(A_{0}, A_{1}, A_{2}\right):=f_{\mathrm{B}}\left(A_{0}\left(\nabla_{1} A_{1} \nabla_{2} A_{2}-\nabla_{2} A_{1} \nabla_{1} A_{2}\right)\right)
$$

for every $A_{0}, A_{1}, A_{2} \in \mathscr{S}_{\mathrm{B}}$. This provides a second class $\left[\Psi_{\mathrm{B}}\right] \in \mathrm{HC}^{\mathrm{even}}\left(\mathscr{S}_{\mathrm{B}}\right)$ and a second formula for the canonical pairing with the K-theory defined by

$$
\mathrm{c}_{\mathrm{B}}([\mathrm{P}]):=\frac{\mathrm{i}}{\ell_{\mathrm{B}}^{2}}\left\langle\left[\Psi_{\mathrm{B}}\right],[\mathrm{P}]\right\rangle=\frac{\mathrm{i}}{\ell_{\mathrm{B}}^{2}} \Psi_{\mathrm{B}}(\mathrm{P}, \mathrm{P}, \mathrm{P}), \quad[\mathrm{P}] \in \mathrm{K}_{0}\left(\mathscr{S}_{\mathrm{B}}\right)
$$


where $\mathrm{P} \in \mathscr{S}_{\mathrm{B}}$ is any representative of $[\mathrm{P}]$ inside the algebra. The map $\mathrm{c}_{\mathrm{B}}$ provides the Chern number of the class $[\mathrm{P}]$ (or of the projection $\mathrm{P}$ with a little abuse of terminology) and defines a second group isomorphism

$$
\mathrm{c}_{\mathrm{B}}: \mathrm{K}_{\mathrm{O}}\left(\mathscr{S}_{\mathrm{B}}\right) \stackrel{\simeq}{\longrightarrow} \mathbb{Z}
$$

generated by $c_{B}\left(\Pi_{0}\right)=1$ [DGM, Section 3.7]. Again, the integrality of the map $c_{B}$ above can be seen as a special case of [Xia, Theorem 3.3] when the hull of the potentials collapses to a singleton. In view of $\mathrm{HC}^{\text {even }}\left(\mathscr{S}_{\mathrm{B}}\right) \simeq \mathbb{Z}$ (Lemma B.1) one infers that $\left[f_{\mathrm{B}}\right]=\left[\Psi_{\mathrm{B}}\right]$ and therefore one has the equality

$$
\mathrm{gl}_{\mathrm{B}}([\mathrm{P}])=\mathrm{c}_{\mathrm{B}}([\mathrm{P}]), \quad \forall[\mathrm{P}] \in \mathrm{K}_{0}\left(\mathscr{S}_{\mathrm{B}}\right) .
$$

The maps (1.14) and (1.17) have important physical manings in the context of the geometric interpretation of the Quantum Hall Effect [Bel1, Xia, BES]. Let $\mathrm{H}$ be a possibly unbounded self-adjoint operator affiliated to $\mathscr{M}_{\mathrm{B}}$. Assume that the spectrum of $\mathrm{H}$ is bounded from below, and for every (Fermi) energy $E \in \rho(H)$ in the resolvent set of $H$, the spectral projection $\mathrm{P}_{\mathrm{E}}:=\chi_{(-\infty, \mathrm{E})}(\mathrm{H})$ lies in the pre- $\mathrm{C}^{*}$ algebra $\mathscr{S}_{\mathrm{B}}$. In this case

$$
\mathrm{N}_{\mathrm{H}}(\mathrm{E}):=\frac{1}{2 \pi \ell_{\mathrm{B}}^{2}} g \mathrm{l}_{\mathrm{B}}\left(\left[\mathrm{P}_{\mathrm{E}}\right]\right)
$$

provides the integrated density of states of $\mathrm{H}$ inside the spectral gap detected by $\mathrm{E}$ [Ves] and

$$
\sigma_{H}(E):=\frac{e^{2}}{2 \pi \hbar} c_{B}\left(\left[P_{E}\right]\right)
$$

is the Hall conductance associated to the energy spectrum of $\mathrm{H}$ below the (Fermi) energy $E$ (the prefactor has the physical units of a conductance). For instance, the results above apply to the Landau Hamiltonian $\mathrm{H}_{\mathrm{B}}$ given by (1.6) since the Landau projections $\Pi_{j}$ are in $\mathscr{S}_{\mathrm{B}}$. In this context the equality (1.18) is known as Strěda formula.

1.2. New results. The main novelty of this work is to reformulate the results presented in the previous section, and in particular the integrality of the maps (1.14) and (1.17), in the context of the geometry of the magnetic spectral triple $\left(\mathscr{S}_{\mathrm{B}}, \mathcal{H}_{4}, \mathrm{D}_{\mathrm{B}}\right)$ introduced in [DS]. The latter is defined by the Hilbert space

$$
\mathcal{H}_{4}:=\mathrm{L}^{2}\left(\mathbb{R}^{2}\right) \otimes \mathbb{C}^{4},
$$

on which the von Neumann algebra $\mathscr{M}_{\mathrm{B}}$, along with each of its subalgebras like $\mathscr{S}_{\mathrm{B}}$, are represented diagonally, i.e.

$$
\pi: A \longmapsto A \otimes \mathbf{1}_{4}=\left(\begin{array}{cccc}
A & 0 & 0 & 0 \\
0 & A & 0 & 0 \\
0 & 0 & A & 0 \\
0 & 0 & 0 & A
\end{array}\right), \quad A \in \mathscr{M}_{\mathrm{B}} .
$$


The magnetic Dirac operator is defined by

$$
D_{B}:=\frac{1}{\sqrt{2}}\left(K_{1} \otimes \gamma_{1}+K_{2} \otimes \gamma_{2}+G_{1} \otimes \gamma_{3}+G_{2} \otimes \gamma_{4}\right)
$$

where $\mathrm{K}_{1}$ and $\mathrm{K}_{2}$ are the magnetic momenta (1.7), and $\mathrm{G}_{1}$ and $\mathrm{G}_{2}$ are the dual magnetic momenta given by

$$
\mathrm{G}_{1}=-\mathrm{i} \ell_{\mathrm{B}} \frac{\partial}{\partial x_{2}}-\frac{1}{2 \ell_{\mathrm{B}}} \mathrm{x}_{1}, \quad \mathrm{G}_{2}=-\mathrm{i} \ell_{\mathrm{B}} \frac{\partial}{\partial \mathrm{x}_{1}}+\frac{1}{2 \ell_{\mathrm{B}}} \mathrm{x}_{2}
$$

and $\gamma_{1}, \ldots, \gamma_{4}$ is any set of Hermitian $4 \times 4$ matrices which satisfy the fundamental anticommutation relations of the Clifford algebra $\mathrm{C}_{4}(\mathbb{C})$. Without loss of generality will fix the following convenient choice ${ }^{1}$ :

$$
\begin{aligned}
& \gamma_{1}:=\left(\begin{array}{cccc}
0 & 0 & 0 & 1 \\
0 & 0 & 1 & 0 \\
0 & 1 & 0 & 0 \\
1 & 0 & 0 & 0
\end{array}\right), \quad \gamma_{2}:=\left(\begin{array}{cccc}
0 & 0 & 0 & \mathrm{i} \\
0 & 0 & \mathrm{i} & 0 \\
0 & -\mathrm{i} & 0 & 0 \\
-\mathrm{i} & 0 & 0 & 0
\end{array}\right) \text {, } \\
& \gamma_{3}:=\left(\begin{array}{cccc}
0 & -1 & 0 & 0 \\
-1 & 0 & 0 & 0 \\
0 & 0 & 0 & 1 \\
0 & 0 & 1 & 0
\end{array}\right), \quad \gamma_{4}:=\left(\begin{array}{cccc}
0 & -\mathrm{i} & 0 & 0 \\
\mathrm{i} & 0 & 0 & 0 \\
0 & 0 & 0 & -\mathrm{i} \\
0 & 0 & \mathrm{i} & 0
\end{array}\right) \text {. }
\end{aligned}
$$

The magnetic Dirac operator is essentially self-adjoint on the dense domain $S\left(\mathbb{R}^{2}\right) \otimes \mathbb{C}^{4}$ and has compact resolvent [DS, Proposition 3.1]. By a straightforward computation one gets

$$
\mathrm{D}_{\mathrm{B}}^{2}:=\left(\begin{array}{cccc}
\mathrm{Q}_{\mathrm{B}} & 0 & 0 & 0 \\
0 & \mathrm{Q}_{\mathrm{B}} & 0 & 0 \\
0 & 0 & \mathrm{Q}_{\mathrm{B}} & 0 \\
0 & 0 & 0 & \mathrm{Q}_{\mathrm{B}}
\end{array}\right)+\left(\begin{array}{cccc}
-\mathbf{1} & 0 & 0 & 0 \\
0 & 0 & 0 & 0 \\
0 & 0 & \mathbf{1} & 0 \\
0 & 0 & 0 & 0
\end{array}\right)
$$

where the operator

$$
\mathrm{Q}_{\mathrm{B}}:=\frac{1}{2}\left(\mathrm{~K}_{1}^{2}+\mathrm{K}_{2}^{2}+\mathrm{G}_{1}^{2}+\mathrm{G}_{2}^{2}\right)
$$

is the two-dimensional isotropic harmonic oscillator on $\mathrm{L}^{2}\left(\mathbb{R}^{2}\right)$. The latter is diagonalized on the Laguerre basis according to

$$
\mathrm{Q}_{\mathrm{B}} \psi_{\mathrm{n}, \mathrm{m}}=(\mathrm{n}+\mathrm{m}+1) \psi_{\mathrm{n}, \mathrm{m}}, \quad(\mathrm{n}, \mathrm{m}) \in \mathbb{N}_{0}^{2} \text {. }
$$

\footnotetext{
${ }^{1}$ It is worth noting that the definition of the $\gamma$-matrices differs from that in [DS, p. 31]. However the two set of $\gamma$-matrices are related by the unitary involution
}

$$
I:=\left(\begin{array}{llll}
0 & 0 & 0 & 1 \\
0 & 1 & 0 & 0 \\
0 & 0 & 1 & 0 \\
1 & 0 & 0 & 0
\end{array}\right) .
$$


As a consequence $Q_{\text {в }}$ has a pure point positive spectrum with eigenvalues $\lambda_{j}:=j+1$, $j \in \mathbb{N}_{0}$, of finite multiplicity $\operatorname{Mult}\left[\lambda_{j}\right]=j+1$. The operator $D_{B}^{2}$ has a simple zero eigenvalue and therefore it is not invertible. For this reason we need to introduce the regularized inverse powers

$$
\left|\mathrm{D}_{\mathrm{B}, \varepsilon}\right|^{-\mathrm{s}}:=\left(\mathrm{D}_{\mathrm{B}}^{2}+\varepsilon \mathbf{I}\right)^{-\frac{s}{2}}, \quad \varepsilon>0, \quad \mathrm{~s} \geqslant 1 .
$$

The last ingredient we need to describe our first result is the Dixmier trace $\operatorname{Tr}_{\text {Dix }}$. There are several standard references for the theory of the Dixmier trace, like [Con, Chap. 4, Sect. 2], [CM, Appendix A], [GVF, Sect. 7.5 and App. 7.C], [LSZ], [AM], and we will refer to these sources for the construction and the properties of the Dixmier trace. A brief summary of the most relevant information can be found in [DGM, Appendix B]. Here, we will fix just few notations (see also Appendix A.1). The domain of definition of the Dixmier trace, called the Dixmier ideal, will be denoted with $\mathfrak{S}^{1^{+}}$. The ideal $\mathfrak{S}_{0}^{1^{+}} \subset \mathfrak{S}^{1^{+}}$ is the closure of the finite-rank operators in the norm of $\mathfrak{S}^{1^{+}}$and every Dixmier trace vanishes on $\mathfrak{S}_{0}^{1+}$. The closed subspace of measurable elements (those for which the Dixmier trace does not depend on the choice of scale-invariant generalized limit) will be denoted with $\mathfrak{S}_{\mathrm{m}}{ }^{+}$. Clearly $\mathfrak{S}_{0}^{1^{+}} \subset \mathfrak{S}_{\mathrm{m}}{ }^{+}$. As proved in [DS, Proposition 2.25], one has that $\left|D_{\mathrm{B}, \varepsilon}\right|^{-4} \in \mathfrak{S}_{\mathrm{m}}^{1^{+}}$and $\operatorname{Tr}_{\mathrm{Dix}}\left(\left|\mathrm{D}_{\mathrm{B}, \varepsilon}\right|^{-4}\right)=2$. However, this integrability property changes considerably when the quantity (1.26) is "dressed" with suitable elements of the magnetic $C^{*}$-algebra. Indeed from [DS, Proposition 2.27] one obtains that

$$
\left|\mathrm{D}_{\mathrm{B}, \varepsilon}\right|^{-2} \pi(\mathrm{A}) \in \mathfrak{S}_{\mathrm{m}}^{1^{+}}, \quad \forall \mathrm{A} \in \mathscr{L}_{\mathrm{B}}^{1} .
$$

Let us introduce the noncommutative integral (a la Connes)

$$
\mathfrak{I n t}_{\mathrm{B}}(A):=\frac{1}{4} \operatorname{Tr}_{\text {Dix }}\left(\left|\mathrm{D}_{\mathrm{B}, \varepsilon}\right|^{-2} \pi(A)\right) .
$$

Then it holds true that [DS, eq. (3.4)]

$$
\mathfrak{I n t}_{\mathrm{B}}(A)=f_{\mathrm{B}}(\mathrm{A}), \quad \forall A \in \mathscr{L}_{\mathrm{B}}^{1}
$$

Equalities (1.29) and (1.12) also provide the proportionality constant between the noncommutative integral $\mathfrak{I n t}_{\mathrm{B}}$ and the trace per unit of volume. Since $\mathscr{S}_{\mathrm{B}} \subset \mathscr{L}_{\mathrm{B}}^{1}$, one infers from (1.27) that the magnetic spectral triple $\left(\mathscr{S}_{\mathrm{B}}, \mathcal{H}_{4}, \mathrm{D}_{\mathrm{B}}\right)$ has spectral dimension 2 as discussed in [DS, Theorem 3.6].

Interestingly, the equality established by (1.28), along with the properties of $f_{B}$, can be used to deduce that the noncommutative integral $\mathfrak{I n t}_{\mathrm{B}}$, as defined by (1.28), is a 0 cocycle of the algebra $\mathscr{S}_{\mathrm{B}}$ which provides a different representative for the class $\left[f_{\mathrm{B}}\right] \in$ $\mathrm{HC} C^{\text {even }}\left(\mathscr{S}_{\mathrm{B}}\right)$. This fact provides a new way of computing the gap labeling function in (1.14) via the noncommutative integral of the spectral triple $\left(\mathscr{S}_{\mathrm{B}}, \mathcal{H}_{4}, \mathrm{D}_{\mathrm{B}}\right)$. 
Theorem 1.1 (Gap labeling). Let $\mathrm{gl}_{\mathrm{B}}: \mathrm{K}_{\mathrm{O}}\left(\mathscr{S}_{\mathrm{B}}\right) \rightarrow \mathbb{Z}$ be the gap labeling isomorphism defined in (1.14). Then, it holds true that

$$
\operatorname{gl}_{\mathrm{B}}([\mathrm{P}])=\mathfrak{I n t}_{\mathrm{B}}(\mathrm{P}), \quad[\mathrm{P}] \in \mathrm{K}_{\mathrm{O}}\left(\mathscr{S}_{\mathrm{B}}\right)
$$

where $\mathrm{P} \in \mathscr{S}_{\mathrm{B}}$ is any representative of the class $[\mathrm{P}]$.

For the description of the second main result we need the operator

$$
\Gamma:=\mathbf{I} \otimes \mathrm{i} \gamma_{1} \gamma_{2}=\left(\begin{array}{cccc}
\mathbf{1} & 0 & 0 & 0 \\
0 & \mathbf{1} & 0 & 0 \\
0 & 0 & -\mathbf{1} & 0 \\
0 & 0 & 0 & -\mathbf{I}
\end{array}\right) .
$$

This is a self-adjoint involution, i.e. $\Gamma=\Gamma^{*}=\Gamma^{-1}$. By combining the Dirac operator $\mathrm{D}_{\mathrm{B}}$ and the involution $\Gamma$ one can define the expression

$$
\mathfrak{C h}_{B}\left(A_{0}, A_{1}, A_{2}\right):=2 \mathfrak{I n t}_{B}\left(\Gamma \pi\left(A_{0}\right)\left[D_{B}, \pi\left(A_{1}\right)\right]\left[D_{B}, \pi\left(A_{2}\right)\right]\right),
$$

where $\mathfrak{I n t}_{B}$ is given by (1.28). It turns out that $\mathfrak{C h}_{B}$ is well-defined on every triple $A_{0}, A_{1}, A_{2} \in \mathscr{S}_{\mathrm{B}}$. More precisely, one has that:

Lemma 1.2 (Second Connes' formula ${ }^{2}$ - version 1). It holds true that

$$
\mathfrak{C h}_{\mathrm{B}}\left(\mathrm{A}_{0}, \mathrm{~A}_{1}, \mathrm{~A}_{2}\right)=\frac{\mathrm{i}}{\ell_{\mathrm{B}}^{2}} \Psi_{\mathrm{B}}\left(\mathrm{A}_{0}, \mathrm{~A}_{1}, \mathrm{~A}_{2}\right), \quad \forall \mathrm{A}_{0}, \mathrm{~A}_{1}, \mathrm{~A}_{2} \in \mathscr{S}_{\mathrm{B}}
$$

with $\Psi_{\mathrm{B}}$ given by (1.15). As a consequence, $\mathfrak{C h}_{\mathrm{B}}$ is a cyclic 2-cocycle of $\mathscr{S}_{\mathrm{B}}$.

The proof of Lemma 1.2 relies on a direct computation and the details are postponed to Section 3. As a direct consequence of Lemma 1.2, one gets that $\mathfrak{C h}_{B}$ provides a different representative for the class $\left[\Psi_{\mathrm{B}}\right] \in \mathrm{HC} C^{\mathrm{even}}\left(\mathscr{S}_{\mathrm{B}}\right)$, up to the right constant. In view of this observation, one can compute the Chern number map (1.17) by using directly the cocycle $\mathfrak{C h}_{\mathrm{B}}$.

Theorem 1.3 (Chern number map). Let $\mathrm{c}_{\mathrm{B}}: \mathrm{K}_{0}\left(\mathscr{S}_{\mathrm{B}}\right) \rightarrow \mathbb{Z}$ be the isomorphism defined in (1.17). Then, it holds true that

$$
\mathrm{c}_{\mathrm{B}}([\mathrm{P}])=\mathfrak{C h}_{\mathrm{B}}(\mathrm{P}, \mathrm{P}, \mathrm{P}), \quad[\mathrm{P}] \in \mathrm{K}_{\mathrm{O}}\left(\mathscr{S}_{\mathrm{B}}\right)
$$

where $\mathrm{P} \in \mathscr{S}_{\mathrm{B}}$ is any representative of the class $[\mathrm{P}]$.

It is worth to point out that the result contained in Theorem 1.3 relates the topology of $\mathscr{S}_{\mathrm{B}}$ with the geometry of the spectral triple $\left(\mathscr{S}_{\mathrm{B}}, \mathcal{H}_{4}, \mathrm{D}_{\mathrm{B}}\right)$.

\footnotetext{
${ }^{2}$ The name second Connes' formula is borrowed from [BES, Theorem 10]. It is worth to point out that the first Connes' formula for the magnetic spectral triple has been proved in [DS].
} 
Remark 1.4 (Involutions and topological triviality). By using the full set of $\gamma$ matrices one can construct the operator

$$
\chi:=\mathbf{I} \otimes \gamma_{1} \gamma_{2} \gamma_{3} \gamma_{4}=\left(\begin{array}{cccc}
-\mathbf{T} & 0 & 0 & 0 \\
0 & +\mathbf{1} & 0 & 0 \\
0 & 0 & -\mathbf{1} & 0 \\
0 & 0 & 0 & +\mathbf{I}
\end{array}\right) .
$$

Like $\Gamma$, this is also an involution, i.e. $\chi=\chi^{*}=\chi^{-1}$. Moreover, $\Gamma$ anti-commutes with the Dirac operator, i.e. $\chi \mathrm{D}_{\mathrm{B}}=-\mathrm{D}_{\mathrm{B}} \chi$. This makes $\left(\mathscr{S}_{\mathrm{B}}, \mathcal{H}_{4}, \mathrm{D}_{\mathrm{B}}, \chi\right)$ an even spectral triple [DS, Section 3.1]. The latter property is not shared by the involution $\Gamma$. In fact, an easy calculation shows that $\Gamma D_{B} \neq-D_{B} \Gamma$ (see Appendix A.3). In view of this consideration, it would seem natural to consider the involution $\chi$ instead $\Gamma$ in the construction of the 2-cocycle (1.32). However, if one defines the quantity

$$
\widehat{\mathfrak{C h}_{\mathrm{B}}}\left(\mathrm{A}_{0}, A_{1}, A_{2}\right):=2 \mathfrak{I n t}_{\mathrm{B}}\left(\chi \pi\left(A_{0}\right)\left[\mathrm{D}_{\mathrm{B}}, \pi\left(A_{1}\right)\right]\left[\mathrm{D}_{\mathrm{B}}, \pi\left(A_{2}\right)\right]\right) \text {, }
$$

then the argument described in Remark 3.1 provides

$$
\widehat{\mathfrak{C h}_{\mathrm{B}}}(\mathrm{P}, \mathrm{P}, \mathrm{P})=0, \quad \forall \mathrm{P} \in \mathscr{S}_{\mathrm{B}} \text {. }
$$

The triviality expressed by equation (1.37) has a deeper motivation. In fact the even spectral triple $\left(\mathscr{S}_{\mathrm{B}}, \mathcal{H}_{4}, \mathrm{D}_{\mathrm{B}}, \chi\right)$ turns out to be a representative of the trivial class in the KK-homology of $\mathscr{S}_{\mathrm{B}}$ [Bou].

Theorem 1.3 suggests the possibility of expressing the Chern number map (1.17) inside the theory of the quantized calculus [Con, Chapter IV] associated with the magnetic spectral triple $\left(\mathscr{S}_{\mathrm{B}}, \mathcal{H}_{4}, \mathrm{D}_{\mathrm{B}}\right)$. However, as suggested by Remark 1.4 it is not the right choice to consider the latter as an even spectral triple with respect to the involution $\chi$. Moreover, definition (1.32) shows that an important role is played by the involution $\Gamma$. All these reasons lead to develop the quantized calculus for the quasi-even (cf. Definition 2.1) magnetic spectral triple $\left(\mathscr{S}_{\mathrm{B}}, \mathcal{H}_{4}, \mathrm{D}_{\mathrm{B}}, \Gamma\right)$. This will be done in full detail in Section 2. In order to anticipate the main results let us introduce the Dirac phase

$$
F_{B, \varepsilon}:=\frac{D_{B}}{\left|D_{B, \varepsilon}\right|}, \quad \varepsilon>0
$$

the quasi-differential (cf. Section 2.2)

$$
\mathrm{d}_{\mathrm{B}} \mathrm{T}:=\left[\mathrm{F}_{\mathrm{B}, \varepsilon}, \mathrm{T}\right]=\mathrm{F}_{\mathrm{B}, \varepsilon} \mathrm{T}-\mathrm{TF}_{\mathrm{B}, \varepsilon},
$$

which, in principle, is well-defined for every bounded operator $\mathrm{T} \in \mathscr{B}\left(\mathcal{H}_{4}\right)$, and the compatible graded trace (cf. Definition 2.6)

$$
\mathfrak{t r}_{\Gamma}(\mathrm{T}):=\operatorname{Tr}_{\text {Dix }}(\Gamma \mathrm{T}),
$$

which is well-defined whenever $T \in \mathfrak{S}_{\mathrm{m}}^{1^{+}}$Then, it follows that the compatible graded trace $\mathfrak{t r}_{\Gamma}$ and the quasi-differential $\mathrm{d}_{\mathrm{B}}$ provide the constitutive elements of a quasi-cycle of dimension 2 for the smooth magnetic algebra $\mathscr{S}_{\mathrm{B}}$. This concept will be clarified in full 
detail in Section 2.3. Associated with the two-dimensional quasi-cycle there is a canonical character defined by

$$
\tau_{\mathrm{B}, 2}\left(A_{0}, A_{1}, A_{2}\right):=\frac{1}{2} \mathfrak{t r}_{\Gamma}\left(\pi\left(A_{0}\right) d_{B} \pi\left(A_{1}\right) d_{B} \pi\left(A_{2}\right)\right),
$$

which turns out to be well-defined for every $A_{0}, A_{1}, A_{2} \in \mathscr{S}_{\mathrm{B}}$. Interestingly, the character $\tau_{\mathrm{B}, 2}$ identifies with $\Psi_{\mathrm{B}}$ as showed in the following result, whose proof is postponed to Section 3.

Lemma 1.5 (Second Connes' formula - version 2). It holds true that

$$
\tau_{\mathrm{B}, 2}\left(A_{0}, A_{1}, A_{2}\right)=\frac{i}{\ell_{\mathrm{B}}^{2}} \Psi_{\mathrm{B}}\left(A_{0}, A_{1}, A_{2}\right), \quad \forall A_{0}, A_{1}, A_{2} \in \mathscr{S}_{\mathrm{B}}
$$

with $\Psi_{\mathrm{B}}$ given by (1.15). As a consequence, $\tau_{\mathrm{B}, 2}$ is a cyclic 2-cocycle of $\mathscr{S}_{\mathrm{B}}$.

Since $\tau_{\mathrm{B}, 2}$ is a cyclic 2-cocycle of $\mathscr{S}_{\mathrm{B}}$, it defines a class in the cyclic cohomology which is usually denoted as $\mathrm{Ch}_{2}\left(\mathcal{H}_{4}, \mathrm{~F}_{\mathrm{B}, \varepsilon}\right):=\left[\tau_{\mathrm{B}, 2}\right] \in \operatorname{HC}^{\text {even }}\left(\mathscr{S}_{\mathrm{B}}\right)$. According to the common use, we will refer to $\mathrm{Ch}_{2}\left(\mathcal{H}_{4}, \mathrm{~F}_{\mathrm{B}, \varepsilon}\right)$ as the Chern character of the quasieven Fredholm module $\left(\mathcal{H}_{4}, \mathrm{~F}_{\mathrm{B}, \varepsilon}\right)$ endowed with the involution $\Gamma$. As a consequence of Lemma 1.5 and Lemma 1.2 one obtains the following restatement of Theorem 1.3.

Theorem 1.6 (Chern character). The isomorphism $\mathrm{c}_{\mathrm{B}}: \mathrm{K}_{0}\left(\mathscr{S}_{\mathrm{B}}\right) \rightarrow \mathbb{Z}$ defined by (1.17) provides the pairing between $\mathrm{K}_{0}\left(\mathscr{S}_{\mathrm{B}}\right)$ and the Chern character $\mathrm{Ch}_{2}\left(\mathcal{H}_{4}, \mathrm{~F}_{\mathrm{B}, \varepsilon}\right)$ of the Fredholm module $\left(\mathcal{H}_{4}, \mathrm{~F}_{\mathrm{B}, \varepsilon}\right)$, i.e.

$$
\mathrm{c}_{\mathrm{B}}([\mathrm{P}])=\left\langle\mathrm{Ch}_{2}\left(\mathcal{H}_{4}, \mathrm{~F}_{\mathrm{B}, \varepsilon}\right),[\mathrm{P}]\right\rangle, \quad[\mathrm{P}] \in \mathrm{K}_{0}\left(\mathscr{S}_{\mathrm{B}}\right) .
$$

Corollary 1.7 (The Connes-Kubo-Chern formula). Let $\mathrm{H}$ be a self-adjoint operator affiliated with the magnetic von Neumann algebra $\mathscr{M}_{\mathrm{B}}$. Assume that the spectrum of $\mathrm{H}$ is bounded from below, and that for every (Fermi) energy $\mathrm{E} \in \rho(\mathrm{H})$ in the resolvent set of $\mathrm{H}$ the spectral projection $\mathrm{P}_{\mathrm{E}}:=\chi_{(-\infty, \mathrm{E})}(\mathrm{H})$ lies in $\mathscr{S}_{\mathrm{B}}$. Then the Hall conductance associated to the energy spectrum of $\mathrm{H}$ below the (Fermi) energy $\mathrm{E}$ is given by

$$
\sigma_{H}(E)=\frac{e^{2}}{2 \pi \hbar}\left\langle\mathrm{Ch}_{2}\left(\mathcal{H}_{4}, \mathrm{~F}_{\mathrm{B}, \varepsilon}\right),\left[\mathrm{P}_{\mathrm{E}}\right]\right\rangle \text {. }
$$

Remark 1.8 (Compatible graded trace and noncommutative integral). It is worth spending some words about a comparison between the noncommutative integral $\mathfrak{I n t}_{\mathrm{B}}$ defined by (1.28) and the compatible graded trace $\mathfrak{t r}_{\Gamma}$ defined by (1.40). Both are built by means of the Dixmier trace $\operatorname{Tr}_{\text {Dix }}$ but in $\mathfrak{I n t}_{B}$ the Dixmier trace is weighted by the term $\left|D_{B, \varepsilon}\right|^{-2}$ which plays the role of a (noncommutative) infinitesimal element of volume. From Lemma 1.2 and Lemma 1.5 one infers the equality $\tau_{\mathrm{B}, 2}=\mathfrak{C h}_{\mathrm{B}}$. However, $\tau_{\mathrm{B}, 2}$ is defined in terms of $\mathfrak{t r}_{\Gamma}$ while $\mathfrak{C h}_{\mathrm{B}}$ is constructed with $\mathfrak{I n t}_{\mathrm{B}}$. Nevertheless the equality between the two 2cocycles is made possible since the quasi-differential $\mathrm{d}_{B}$ which enters in the construction of $\tau_{B, 2}$ provides a weight proportional to $\left|D_{B, \varepsilon}\right|^{-1}$, which is exactly the square root of the infinitesimal element of volume. 
The novelty of the results contained in Theorem 1.1, Theorem 1.3, and Theorem 1.6 consists on the use of the magnetic spectral triple $\left(\mathscr{S}_{\mathrm{B}}, \mathcal{H}_{4}, \mathrm{D}_{\mathrm{B}}\right)$, or in the associated Fredholm module $\left(\mathcal{H}_{4}, \mathrm{~F}_{\mathrm{B}, \varepsilon}\right)$, for the study of the topology of the magnetic algebra $\mathscr{S}_{\mathrm{B}}$. The relevance of this approach relies on the fact that the Dirac operator $\mathrm{D}_{\mathrm{B}}$, as defined by (1.22), has compact resolvent. Equivalently, the Dirac phase $F_{B, \varepsilon}$ is a compact operator. This compactness is the real new insight of our approach to the study of the magnetic algebra, which indeed contrasts with other approaches already present in the literature. For a more precise analysis on this aspect we refer to the long discussion contained in [DS, Section 1] and references therein. In order to advocate for the usefulness of the compactness in our approach, let us rewrite the integrated density of states in (1.19) and the Hall conductance in (1.20) in combination with the results of Theorem 1.1 and Theorem 1.6. By making explicit the role of the Dixmier trace and of the resolvent of $D_{B}$ in the definition of the noncommutative integral $\mathfrak{I n t}_{\mathrm{B}}$, one obtains

$$
\mathrm{N}_{\mathrm{H}}(\mathrm{E})=\frac{1}{8 \pi \ell_{\mathrm{B}}^{2}} \operatorname{Tr}_{\text {Dix }}\left(\left|\mathrm{D}_{\mathrm{B}, \varepsilon}\right|^{-2} \pi\left(\mathrm{P}_{\mathrm{E}}\right)\right)
$$

for the integrated density of states, and

$$
\begin{aligned}
\sigma_{H}(E) & =\frac{e^{2}}{4 \pi \hbar} \operatorname{Tr}_{\text {Dix }}\left(\Gamma \pi\left(P_{E}\right) d_{B} \pi\left(P_{E}\right) d_{B} \pi\left(P_{E}\right)\right) \\
& =\frac{e^{2}}{4 \pi \hbar} \operatorname{Tr}_{\text {Dix }}\left(\left|D_{B, \varepsilon}\right|^{-2} \Gamma \pi\left(P_{E}\right)\left[D_{B}, \pi\left(P_{E}\right)\right]^{2}\right)
\end{aligned}
$$

for the Hall conductance. Since the operator $\left|D_{B, \varepsilon}\right|^{-2}$ is diagonalized by the Laguerre basis $\left\{\psi_{n, m}\right\}$, one can hope to use this natural "discretization" to deduce from (1.44) and (1.45) approximate formulas for $N_{H}(E)$ and $\sigma_{H}(E)$. In the case of tight-binding magnetic operators on $\ell^{2}\left(\mathbb{Z}^{2}\right)$ similar approximated formulas already exists, based on the discreteness of the lattice $\mathbb{Z}^{2}$. In fact the density of states for tight-binding magnetic operators can be estimated with the windowed DOS [LLW] while the Chern numbers can be computed with the spectral localizer formula [LSB1, LSB2, LSB3]. Our guess is that the latter results can be adapted to the magnetic operators on $L^{2}\left(\mathbb{R}^{2}\right)$ on the basis of the formulas (1.44) and (1.45). At the moment, this idea is under investigation.

Structure of the paper. In Section 2.1 we introduce a generalization of an even Fredholm module, these so-called quasi-even Fredholm modules, which will be used to study the differential theory of the magnetic algebra. The interest of this generalization lies in the fact that quotient by a convenient ideal of compact operators gives rise to a genuine even Fredholm module. In Section 2.2 we study the differential theory of quasi-even Fredholm modules, with the goal of defining the notion of a k-cycle over a quasi-even Fredholm modules in Section 2.3. Here we also introduce an appropriate notion of a graded trace compatible with quasi-even Fredholm modules. In Section 2.4 we identify the Chern character of the 2-cycle associated to the magnetic algebra to (1.15) via the second Connes' Formula. Section 3 contains the proofs of the key Lemmas 1.2 and 1.5. 
In Appendix A are collected some technical results used in various parts of the paper. Appendix B is devoted to a brief overview of the cyclic cohomology of the magnetic algebra.

Acknowledgments. GD's research is supported by the grant Fondecyt Regular - 1190204. MS's research is supported by the grant CONICYT-PFCHA Doctorado Nacional 2018 21181868. The authors would like to cordially thank Chris Bourne and Hermann SchulzBaldes for several inspiring discussions.

\section{QUANTIZED CALCULUS OF THE MAGNETIC SPECTRAL TRIPLE}

In this section we will build the quantized calculus (a la Connes) for the algebra $\mathscr{S}_{\mathrm{B}}$ based on the geometry of the magnetic spectral triple $\left(\mathscr{S}_{\mathrm{B}}, \mathcal{H}_{4}, \mathrm{D}_{\mathrm{B}}\right)$. Although the treatment presented below follows quite closely the theory presented in [Con, Chapter IV] we will need to change and generalize some definitions to adapt the general scheme to our case of interest.

2.1. Quasi-even Fredholm module. Let $\mathscr{K}(\mathcal{H})$ be the $C^{*}$-algebra of compact operators on a Hilbert space $\mathcal{H}$. Let $\mathscr{A}$ be a pre-C*-algebra and $\pi: \mathscr{A} \rightarrow \mathscr{B}(\mathcal{H})$ a *-representation. Following [Con, Chapter IV], let us recall that a (compact) Fredholm module over $\mathscr{A}$, denoted $(\mathcal{H}, F)$, is determined by a bounded operator $F$ such that: $\left(F-F^{*}\right) \in \mathscr{K}(\mathcal{H})$ (quasi-self-adjoint); $\left(\mathrm{F}^{2}-\mathbf{I}\right) \in \mathscr{K}(\mathcal{H})$ (quasi-involution) and

$$
[\mathrm{F}, \pi(\mathrm{A})]:=\mathrm{F} \pi(\mathrm{A})-\pi(\mathrm{A}) \mathrm{F} \in \mathscr{K}(\mathcal{H}), \quad \forall \mathrm{A} \in \mathscr{A} .
$$

A graded structure on $\mathcal{H}$ is given by a self-adjoint non-trivial ${ }^{3}$ involution $\Gamma=\Gamma^{*}=\Gamma^{-1}$. A bounded operator $\mathrm{T} \in \mathscr{B}(\mathcal{H})$ has degree 0 with respect to $\Gamma$ if $\Gamma \mathrm{T}=\mathrm{T} \Gamma$, and has degree 1 if $\Gamma \mathrm{T}=-\mathrm{T} \Gamma$. We will denote with $\mathscr{B}(\mathcal{H})_{i}$ the subset of bounded operator of degree $i=0,1$. In order to combine a graded structure with a Fredholm module the basic request is that the representation $\pi$ has to be of degree 0, i. e. $\pi(\mathscr{A}) \subseteq \mathscr{B}(\mathcal{H})_{0}$. Said differently, one requires that

$$
\Gamma \pi(\mathrm{A})-\pi(\mathrm{A}) \Gamma=0, \quad \forall \mathrm{A} \in \mathscr{A} .
$$

A Fredholm module $(\mathcal{H}, F)$ with graded structure $\Gamma$ is called even if $F \in \mathscr{B}(\mathcal{H})_{1}$, i. e. when

$$
\{\Gamma, F\}:=\Gamma F+F \Gamma=0
$$

For our aim, equation (2.1) is not satisfied (cf. Remark 1.4) and for this reason we need to adapt the notion of even Fredholm module.

Definition 2.1 (Quasi-even Fredholm module of dimension k). Let $\mathfrak{Z} \subseteq \mathscr{K}(\mathcal{H})$ be a twosided self-adjoint ideal of $\mathscr{B}(\mathcal{H})$ and $\Gamma$ a non-trivial self-adjoint involution. A Fredholm module $(\mathcal{H}, F)$ over $\mathscr{A}$ is called quasi-even of dimension $\mathrm{k}$ with respect to the pair $(\Gamma, \mathfrak{Z})$ if:

(a) $\left[\mathrm{F}^{2}, \pi\left(\mathrm{A}_{0}\right)\right] \in \mathfrak{Z}$ for every $\mathrm{A}_{0} \in \mathscr{A}$;

\footnotetext{
${ }^{3}$ That is $\Gamma \neq \pm \mathbf{1}$. Equivalently, the spectrum of $\Gamma$ is $\{ \pm 1\}$.
} 
(b) $\Gamma\left[F, \pi\left(A_{0}\right)\right] \Gamma=-\left[F, \pi\left(A_{0}\right)\right]+R\left(A_{0}\right) \Gamma$ such that

$$
\left[F, \pi\left(A_{1}\right)\right] \cdots\left[F, \pi\left(A_{n}\right)\right] R\left(A_{0}\right)\left[F, \pi\left(A_{n+1}\right)\right] \cdots\left[F, \pi\left(A_{k-1}\right)\right] \in \mathfrak{Z},
$$

for every $A_{0}, A_{1}, \ldots, A_{n}, A_{n+1}, \ldots, A_{k-1} \in \mathscr{A}$.

(c) $\left[F, \pi\left(A_{0}\right)\right]\left[F, \pi\left(A_{1}\right)\right] \cdots\left[F, \pi\left(A_{k}\right)\right] \in \mathfrak{Z}$ for every $A_{0}, A_{1}, \ldots, A_{k} \in \mathscr{A}$.

Condition (a) of Definition 2.1 generalizes the requirement $F^{2}=\mathbf{1}$, which is usually assumed in the theory of Fredholm modules (see [DS, Remark 3.13] and references therein). It is immediate to observe that every even Fredholm module meets condition (b) of Definition 2.1 with $R=0$. In this sense Definition 2.1 provides a generalization of the notion of even Fredholm module. From condition (b) one deduces that

$$
\begin{aligned}
& \Gamma\left[F, \pi\left(A_{1}\right)\right] \cdots\left[F, \pi\left(A_{k}\right)\right] \\
& =(-1)^{n}\left[F, \pi\left(A_{1}\right)\right] \cdots\left[F, \pi\left(A_{n}\right)\right] \Gamma\left[F, \pi\left(A_{n+1}\right)\right] \cdots\left[F, \pi\left(A_{k}\right)\right]+\mathfrak{Z}
\end{aligned}
$$

for all $A_{1}, \ldots, A_{k} \in \mathscr{A}$. When $n=k$ this implies

$$
\left[\mathrm{F}, \pi\left(A_{1}\right)\right] \cdots\left[\mathrm{F}, \pi\left(A_{\mathrm{k}}\right)\right] \in \mathscr{B}(\mathcal{H})_{\mathrm{k} \bmod 2}+\mathfrak{Z}
$$

Condition (c) stipulates that

$$
\left[F, \pi\left(A_{1}\right)\right] \cdots\left[F, \pi\left(A_{k^{\prime}}\right)\right] \in \mathfrak{Z}
$$

as soon as $k^{\prime}>k$ since $\mathfrak{Z}$ is an ideal.

Now, let us focus on the magnetic Fredholm module $\left(\mathcal{H}_{4}, \mathrm{~F}_{\mathrm{B}, \varepsilon}\right)$ over the pre- $\mathrm{C}^{*}$-algebra $\mathscr{S}_{\mathrm{B}}$, where the Hilbert space $\mathcal{H}_{4}$ is defined by (1.21) and the Dirac phase is defined by (1.38). Let $\Gamma$ be the self-adjoint involution defined by (1.31). Finally, let us recall the notations $\mathfrak{S}^{\mathfrak{p}}$ and $\mathfrak{S}^{\mathfrak{p}^{ \pm}}$for the $p$-th Schatten ideal and for the $p$-th Dixmier/Mačaev ideal, respectively (cf. Appendix A.1). The main properties of the magnetic Fredholm module $\left(\mathcal{H}_{4}, \mathrm{~F}_{\mathrm{B}, \varepsilon}\right)$ are contained in the following result.

Proposition 2.2. The following facts hold true:

(1) $\left[\mathrm{F}_{\mathrm{B}, \varepsilon}, \pi(\mathrm{A})\right] \in \mathfrak{S}^{2^{+}}$for every $\mathrm{A} \in \mathscr{S}_{\mathrm{B}}$;

(2) $\left[\mathrm{F}_{\mathrm{B}, \varepsilon}^{2}, \pi(\mathrm{A})\right] \in \mathfrak{S}^{1}$ for every $\mathrm{A} \in \mathscr{S}_{\mathrm{B}}$;

(3) Let $R\left(A_{0}\right):=\Gamma\left[F_{B, \varepsilon}, \pi\left(A_{0}\right)\right] \Gamma+\left[F_{B, \varepsilon}, \pi\left(A_{0}\right)\right]$, then

$$
\mathrm{R}\left(\mathrm{A}_{0}\right)\left[\mathrm{F}_{\mathrm{B}, \varepsilon}, \pi\left(\mathrm{A}_{1}\right)\right] \in \mathfrak{S}^{1}, \quad\left[\mathrm{~F}_{\mathrm{B}, \varepsilon}, \pi\left(\mathrm{A}_{1}\right)\right] \mathrm{R}\left(\mathrm{A}_{0}\right) \in \mathfrak{S}^{1}
$$

for every $A_{0}, A_{1} \in \mathscr{S}_{\mathrm{B}}$

(4) $\left[F_{B, \varepsilon}, \pi\left(A_{0}\right)\right]\left[F_{B, \varepsilon}, \pi\left(A_{1}\right)\right]\left[F_{B, \varepsilon}, \pi\left(A_{2}\right)\right] \in \mathfrak{S}^{1}$ for every $A_{0}, A_{1}, A_{2} \in \mathscr{S}_{B}$;

Proof. Item (1) is proved in [DS, Lemma 3.10]. Item (2) follows from the direct computation

$$
\mathrm{F}_{\mathrm{B}, \varepsilon}^{2}-\mathbf{I}=-\varepsilon\left|\mathrm{D}_{\mathrm{B}, \varepsilon}\right|^{-2}
$$


which provides

$$
\left[\mathrm{F}_{\mathrm{B}, \varepsilon}^{2}, \pi(A)\right]=-\varepsilon\left[\left|\mathrm{D}_{\mathrm{B}, \varepsilon}\right|^{-2}, \pi(A)\right]=-\varepsilon \sum_{j=1}^{4}\left[\mathrm{Q}_{\mathrm{B}, \varepsilon_{j}}^{-1}, A\right] \otimes \tau_{j, j}
$$

where $\mathrm{Q}_{\mathrm{B}, \varepsilon_{\mathfrak{j}}}:=\mathrm{Q}_{\mathrm{B}}+\varepsilon_{\mathbf{j}} \mathbf{I}$ with $\mathrm{Q}_{\mathrm{B}}$ the harmonic oscillator (1.25),

$$
\varepsilon_{1}=\varepsilon_{2}=\varepsilon, \quad \varepsilon_{3}=\varepsilon+1, \quad \varepsilon_{4}=\varepsilon-1,
$$

and $\tau_{i, j} \in \operatorname{Mat}_{4}(\mathbb{C})$ the matrix which has a single 1 in the entry at the position $(i, j)$ and zeroes in all other positions. Therefore, to prove the result it is enough to shows that $\left[Q_{B, \varepsilon_{j}}^{-1}, A\right] \in \mathfrak{S}^{1}$ for every $\mathfrak{j}=1, \ldots, 4$ and this is done in Lemma A.2 and Remark A.3. Item (3) follows from Lemma A.6 which shows that $R\left(A_{0}\right) \in \mathfrak{S}^{2^{-}}$. Since $\mathfrak{S}^{2^{+}}$is the dual of $\mathfrak{S}^{2^{-}}$one gets from item (1) and Corollary A.5 the desired result. For item (4) one needs to use the Hölder type inequality for weak Schatten ideals $\mathfrak{S}_{\mathrm{w}}^{\mathrm{p}}$ (see Appendix A.1). since $\left[F_{B, \varepsilon}, \pi\left(A_{j}\right)\right] \in \mathfrak{S}^{2^{+}}=\mathfrak{S}_{\mathrm{w}}^{2}$ one obtains that triple products of these terms lie inside $\mathfrak{S}_{\mathrm{W}}^{\frac{2}{3}}$. The inclusion $\mathfrak{S}_{\mathrm{W}}^{\frac{2}{3}} \subset \mathfrak{S}^{1}$ concludes the proof.

Item (1) of Proposition 2.2 says that the magnetic Fredholm module $\left(\mathcal{H}_{4}, \mathrm{~F}_{\mathrm{B}, \varepsilon}\right)$ is (densely) $2^{+}$-summable (cf. [DS, Theorem 3.12]). Summarizing all the previous results we can state that:

Theorem 2.3. The magnetic Fredholm module $\left(\mathcal{H}_{4}, \mathrm{~F}_{\mathrm{B}, \varepsilon}\right)$ over the pre-C*-algebra $\mathscr{S}_{\mathrm{B}}$ is (densely) $2^{+}$-summable and quasi-even of rank 2 with respect to the pair $\left(\Gamma, \mathfrak{S}^{1}\right)$.

2.2. Quasi-differential structure. Let $(\mathcal{H}, F)$ be a Fredholm module over $\mathscr{A}$ with a quasi-even structure of dimension $k$ with respect to $(\Gamma, \mathfrak{Z})$. Let

$$
\Omega^{0}:=\pi(\mathscr{A})^{+}=\{\pi(A)+c \mathbf{1} \in \mathscr{B}(\mathcal{H}) \mid A \in \mathscr{A}, \quad c \in \mathbb{C}\} .
$$

Observe that $\Omega^{0}=\pi(\mathscr{A})$ whenever $\mathscr{A}$ is unital and $\pi(\mathbf{1})=\mathbf{1}$. Moreover, $\Omega_{\mathrm{B}}^{0}$ is made by element of degree 0 with respect to $\Gamma$, i. e. $\Omega^{0} \subseteq \mathscr{B}(\mathcal{H})_{0}$. The quasi-differential on $\Omega^{0}$ is defined by

$$
\mathrm{d}(\pi(\mathrm{A})+\mathrm{c} \mathbf{1}):=[\mathrm{F}, \pi(\mathrm{A})]
$$

for every $A \in \mathscr{A}$. For $n \in \mathbb{N}$ one lets $\Omega^{n}$ be the linear span of elements of the type

$$
\omega:=\left(\pi\left(A_{0}\right)+c \boldsymbol{1}\right) \mathrm{d} \pi\left(A_{1}\right) \cdots \mathrm{d} \pi\left(A_{n}\right), \quad A_{0}, A_{1}, \ldots, A_{n} \in \mathscr{A} .
$$

In short, one can write $\Omega^{n}:=\pi(\mathscr{A})^{+} \otimes \mathrm{d} \pi(\mathscr{A}) \otimes \ldots \otimes \mathrm{d} \pi(\mathscr{A})$ where the product is repeated $n$-times. From (2.2) it follows that

$$
\Omega^{\mathrm{n}} \subseteq \mathscr{B}(\mathcal{H})_{\mathrm{n} \bmod 2}+\mathfrak{Z}, \quad \mathrm{n} \in \mathbb{N}_{0}
$$

and (2.3) implies that

$$
\Omega^{\mathrm{n}} \subseteq \mathfrak{Z}, \quad \forall \mathrm{n}>\mathrm{k}
$$


The full graded quasi-differential algebra associated with $\mathscr{A}$ is defined as

$$
\Omega^{\bullet}:=\bigoplus_{n \in \mathbb{N}_{0}} \Omega^{n}
$$

In $\Omega_{\mathrm{B}}^{\bullet}$, the product of operators provides a well-defined graded product. More precisely, for every pair of elements $\omega \in \Omega^{n}$ and $\omega^{\prime} \in \Omega^{n^{\prime}}$ one has that $\omega \omega^{\prime} \in \Omega^{n+n^{\prime}}$. The proof of this fact is straightforward (see [Con, Section IV.1]) and is based on the identity

$$
\left[F, \pi\left(A_{1}\right)\right] \pi\left(A_{2}\right)=\left[F, \pi\left(A_{1} A_{2}\right)\right]-\pi\left(A_{1}\right)\left[F, \pi\left(A_{2}\right)\right]
$$

valid for every $A_{1}, A_{2} \in \mathscr{A}$.

The quasi-differential (2.7) can be extended to a map $\mathrm{d}: \Omega^{\bullet} \rightarrow \Omega^{\bullet}$ as follows

$$
\mathrm{d} \omega:=\mathrm{F} \omega-(-1)^{\mathrm{n}} \omega \mathrm{F}, \quad \forall \omega \in \Omega^{\mathrm{n}} .
$$

the main properties of the quasi-differential are listed below.

Proposition 2.4. Let $(\mathcal{H}, F)$ be a Fredholm module over $\mathscr{A}$ with a quasi-even structure of dimension $\mathrm{k}$ with respect to $(\Gamma, \mathfrak{Z})$. The following facts hold true:

(1) $\mathrm{d} \omega \in \Omega^{\mathrm{n}+1}+\mathfrak{Z}$ for every $\omega \in \Omega^{\mathrm{n}}$;

(2) $d^{2} \omega:=d(d \omega) \in \mathfrak{Z}$ for every $\omega \in \Omega^{n}$;

(3) $d\left(\omega_{1} \omega_{2}\right)=\left(d \omega_{1}\right) \omega_{2}+(-1)^{n_{1}} \omega_{1}\left(d \omega_{2}\right)$ for every $\omega_{j} \in \Omega^{n_{j}}$, with $j=1,2$.

Proof. Item (1) follows from the identity

$$
[F, \pi(A)] F=-F[F, \pi(A)]+Z_{A}
$$

where $Z_{A}:=\left[F^{2}, \pi(A)\right] \in \mathfrak{Z}$ by assumption. Therefore, for every $A_{1}, \ldots, A_{n} \in \mathscr{A}$ one gets

$$
\left(\left[F, \pi\left(A_{1}\right)\right] \ldots\left[F, \pi\left(A_{n}\right)\right]\right) F=(-1)^{n} F\left(\left[F, \pi\left(A_{1}\right)\right] \ldots\left[F, \pi\left(A_{n}\right)\right]\right)+Z
$$

for a certain $Z \in \mathfrak{Z}$ which depends on $A_{1}, \ldots, A_{n}$. Let

$$
\eta:=\left(\pi\left(A_{0}\right)+c \boldsymbol{1}\right) \mathrm{d} \pi\left(A_{1}\right) \ldots \mathrm{d} \pi\left(A_{n}\right)
$$

be one of the elemental generators of $\Omega^{n}$. Then, it turns out that

$$
\eta F-(-1)^{n} F \eta=\left[F, \pi\left(A_{0}\right)+c \mathbf{1}\right] d \pi\left(A_{1}\right) \ldots d \pi\left(A_{n}\right)+Z^{\prime}
$$

with $Z^{\prime} \in \mathfrak{Z}$. This, proves that $d \eta \in \Omega^{n+1}+\mathfrak{J}$. Since every $\omega \in \Omega^{n}$ is a linear combination of elements of the type of $\eta$, the result follows by linearity. Item (2) follows by a direct computation which shows that

$$
\mathrm{d}^{2} \omega=\left[\mathrm{F}^{2}, \omega\right], \quad \forall \omega \in \Omega^{\mathrm{n}} .
$$

If $\omega \in \Omega^{0}$ then $\mathrm{d}^{2} \omega \in \mathfrak{Z}$ just by assumption. To complete the proof one can use induction on the order $n$. Let us assume that item (2) is true up to order $n-1$ and consider an element $\eta \in \Omega^{n}$ defined as in (2.8). One has that $\eta=\left(\pi\left(A_{0}\right)+\mathbf{c} \mathbf{1}\right) \eta_{0}$ where 


$$
\begin{aligned}
\eta_{0}:=\mathrm{d} \pi\left(A_{1}\right) & \ldots \mathrm{d} \pi\left(A_{n}\right) \in \Omega^{n-1} \text { Therefore, } \\
& \mathrm{d}^{2} \eta=\left[\mathrm{F}^{2}, \pi\left(\mathrm{A}_{0}\right)\right] \eta_{0}+\left(\pi\left(\mathrm{A}_{0}\right)+\mathrm{c} \mathbf{\mathbf { I }}\right)\left[\mathrm{F}^{2}, \eta_{0}\right] \in \mathfrak{Z}
\end{aligned}
$$

since both $\left[F^{2}, \pi\left(A_{0}\right)\right]$ and $\left[F^{2}, \eta_{0}\right]$ are in $\mathfrak{Z}$ by assumption. By linearity one gets the result for a generic $\omega \in \Omega^{\mathrm{n}}$. The proof of Item (3) amounts to a direct computation.

From items (1) and (2) of Proposition 2.4 one infers that the quasi-differential $\mathrm{d}$ acts on the graded algebra $\Omega^{\bullet}$ as follows:

$$
\begin{aligned}
\mathrm{d}: \Omega^{\mathrm{n}} \longrightarrow \Omega^{\mathrm{n}+1}+\mathfrak{Z}, \quad \forall \mathrm{n} \in \mathbb{N}_{0} . \\
\mathrm{d}^{2}: \Omega^{\mathrm{n}} \longrightarrow 3
\end{aligned}
$$

Moreover, item (3) shows that $\mathrm{d}$ is a graded derivation, i. e. it satisfies a graded version of the Leibniz's rule.

Remark 2.5 (Induced differential structure). Consider the quotient space $\widetilde{\Omega}^{n}:=\Omega^{n} / \mathfrak{Z}$ and the related graded algebra

$$
\widetilde{\Omega}^{\bullet}:=\bigoplus_{n \in \mathbb{N}_{0}} \widetilde{\Omega}^{n}
$$

From Proposition 2.4 one infers that the quasi-differential $\mathrm{d}$ behaves well with respect to the quotient an defines a map

$$
\tilde{\mathrm{d}}: \widetilde{\Omega}^{\mathrm{n}} \longrightarrow \widetilde{\Omega}^{\mathrm{n}+1}, \quad \forall \mathrm{n} \in \mathbb{N}_{0}
$$

such that $\tilde{\mathrm{d}}^{2}=0$. Said differently, the pair $(\widetilde{\Omega} \bullet, \tilde{\mathrm{d}})$ defines a genuine graded differential algebra in the sense described in [Con, Section IV.1]. It is worth noting that according to Definition 2.1 one has that $\widetilde{\Omega}^{n}=0$ for every $n>k$ in the case of a quasi-even structure of dimension $k$.

Let us now focus on the specific case of the magnetic Fredholm module $\left(\mathcal{H}_{4}, \mathrm{~F}_{\mathrm{B}, \varepsilon}\right)$. We will denote with $d_{B}$ the quasi-differential associated with $F_{B, \varepsilon}$ according to the definition (1.39). The related graded quasi-differential algebra associated with the magnetic algebra $\mathscr{S}_{\mathrm{B}}$ will be denoted with

$$
\Omega_{\mathrm{B}}^{\bullet}:=\bigoplus_{n \in \mathbb{N}_{0}} \Omega_{\mathrm{B}}^{\mathrm{n}} \text {. }
$$

As a consequence of Proposition 2.2 one has that $\Omega_{\mathrm{B}}^{1} \subseteq \mathfrak{S}^{2^{+}}$and $\Omega_{\mathrm{B}}^{2} \subseteq \mathfrak{S}^{1^{+}}$. Moreover $\Omega_{\mathrm{B}}^{2} \subseteq \mathscr{B}\left(\mathcal{H}_{4}\right)_{0}+\mathfrak{S}^{1}$, i. e. the elements of $\Omega_{\mathrm{B}}^{2}$ are of degree 0 with respect to $\Gamma$ up to a remainder which is trace class.

2.3. Quasi-cycles. Let $(\mathcal{H}, F)$ be a Fredholm module over $\mathscr{A}$ with a quasi-even structure of dimension $k$ with respect to $(\Gamma, \mathfrak{Z})$. We need to consider a linear map

$$
\mathfrak{t r}_{\Gamma}: \Omega^{k} \longrightarrow \mathbb{C}
$$

which satisfies some relevant conditions. 
Definition 2.6 (A compatible graded trace). Let $(\mathcal{H}, F)$ be a Fredholm module over $\mathscr{A}$ with a quasi-even structure of dimension $k$ with respect to $(\Gamma, \mathfrak{Z})$. A compatible graded trace for $(\mathcal{H}, F)$ is a map like (2.9) such that :

(a) $\mathfrak{Z} \subseteq \operatorname{Ker}\left(\mathfrak{t r}_{\Gamma}\right)$;

(b) $\mathfrak{t r}_{\Gamma}(\mathrm{d}(\omega))=0$ for every $\omega \in \Omega^{\mathrm{k}-1}$;

(c) $\mathfrak{t r}_{\Gamma}\left(\omega_{1} \omega_{2}\right)=(-1)^{\mathfrak{n}_{1} n_{2}} \mathfrak{t r}_{\Gamma}\left(\omega_{2} \omega_{1}\right)$ for every $\omega_{1} \in \Omega^{n_{1}}$ and $\omega_{2} \in \Omega^{n_{2}}$ such that $n_{1}+n_{2}=k$.

Property (b) is the closedness condition of the trace $\mathfrak{t r}_{\Gamma}$ with respect to the quasidifferential d. Property (c) implements the compatibility of $\mathfrak{t r}_{\Gamma}$ with respect to the graded structure of $\Omega^{\bullet}$. Finally, from (2.6) one infers that $\mathfrak{t r}_{\Gamma}\left(\Omega^{\mathfrak{n}}\right)=\{0\}$ for every $n>k$.

The following definition generalizes the concept of cycle given in [Con, Chapter 3, Section 1. $\alpha$ ] or [GVF, Definition 8.3].

Definition 2.7 (Quasi-cycle of dimension k). Let $(\mathcal{H}, F)$ be a Fredholm module over $\mathscr{A}$ with a quasi-even structure of dimension $k$ with respect to $(\Gamma, \mathfrak{Z})$. Let $\Omega^{\bullet}$ be the associated graded quasi-differential algebra with quasi-differential $\mathrm{d}$ induced by $\mathrm{F}$ and $\mathfrak{t r}_{\Gamma}$ a compatible graded trace. Then the triple $\left(\Omega^{\bullet}, \mathrm{d}, \mathfrak{t r}_{\Gamma}\right)$ will be called a quasi-cycle of dimension k over $\mathscr{A}$.

Remark 2.8 (Induced cycle). In the same spirit of Remark 2.5 one can observe that a compatible graded trace behaves well with respect to the quotient with respect to $\mathfrak{Z}$ and defines a trace

$$
\widetilde{\mathfrak{t r}}_{\Gamma}: \widetilde{\Omega}^{k} \longrightarrow \mathbb{C} \text {. }
$$

In particular, one can check that the triple $\left(\widetilde{\Omega} \bullet, \tilde{\mathrm{d}}, \widetilde{\mathfrak{t r}}_{\Gamma}\right)$ defines a genuine cycle of dimension $k$ in the sense of [Con, Chap. 3, Sect. 1. $\alpha$ ] or [GVF, Def. 8.3 \& Def. 8.17].

Now, let us focus on the case of the magnetic Fredholm module $\left(\mathcal{H}_{4}, \mathrm{~F}_{\mathrm{B}, \varepsilon}\right)$. In such a case, a natural candidate for a compatible graded trace is the Dixmier trace. In order to take care of the grading, we will define the compatible graded trace as in (1.40), i. e.

$$
\mathfrak{t r}_{\Gamma}(\omega):=\operatorname{Tr}_{\text {Dix }}(\Gamma \omega), \quad \forall \omega \in \Omega_{\mathrm{B}}^{2} .
$$

It is worth pointing out that there is no need to specify the dependence of the Dixmier trace on the choice of a scale-invariant state. In fact, as commented in [DS, Remark 3.11] one can show that $\Omega_{\mathrm{B}}^{2} \subseteq \mathfrak{S}_{\mathrm{m}}^{1^{+}}$where $\mathfrak{S}_{\mathrm{m}}^{1^{+}} \subset \mathfrak{S}^{1^{+}}$denotes the closed space of measurable elements whose Dixmier trace does not depend on the choice of any scaleinvariant generalized limit. Let us introduce the subspace $\mathfrak{S}_{\mathrm{m}}^{1^{+}}$of element with a vanishing Dixmier trace

$$
\operatorname{Ker}\left(\operatorname{Tr}_{\text {Dix }}\right):=\left\{\mathrm{T} \in \mathfrak{S}_{\mathrm{m}}^{1^{+}} \mid \operatorname{Tr}_{\text {Dix }}(\mathrm{T})=0\right\} .
$$

As discussed in Appendix A.1, one has that $\mathfrak{S}^{1} \subset \mathfrak{S}_{0}^{1^{+}} \subset \operatorname{Ker}\left(\operatorname{Tr}_{\text {Dix }}\right)$. 
Proposition 2.9. The map (2.10) is a compatible graded trace for the magnetic Fredholm module $\left(\mathcal{H}_{4}, \mathrm{~F}_{\mathrm{B}, \varepsilon}\right)$.

Proof. Property (a) of Definition 2.6 is satisfied since the ideal $\mathfrak{S}^{1}$ of trace-class operators is contained in $\operatorname{Ker}\left(\operatorname{Tr}_{\text {Dix }}\right)$. To prove property (b) of Definition 2.6 let us start with an element $\omega=\left(\pi\left(A_{0}\right)+c \mathbf{I}\right) \mathrm{d}_{\mathrm{B}} \pi\left(A_{1}\right) \in \Omega^{1}$. Then $\mathrm{d}_{\mathrm{B}} \omega=\mathrm{d}_{\mathrm{B}} \pi\left(A_{0}\right) \mathrm{d}_{\mathrm{B}} \pi\left(A_{1}\right)+Z$ with $\mathrm{Z} \in \mathfrak{S}^{1}$ and in turn

$$
\mathfrak{t r}_{\Gamma}\left(\mathrm{d}_{\mathrm{B}} \mathrm{w}\right)=\mathfrak{t r}_{\Gamma}\left(\left[\mathrm{F}_{\mathrm{B}, \varepsilon}, \pi\left(A_{0}\right)\right]\left[\mathrm{F}_{\mathrm{B}, \varepsilon}, \pi\left(A_{1}\right)\right]\right)=0
$$

in view of Corollary 3.3. The general case follows by linearity since $\Omega^{1}$ is the linear span of elements of the form of $\omega$. Property (c) of Definition 2.6 can be shown with a direct computation. There are two possible cases. Let us start with the case $\omega_{0} \in \Omega_{\mathrm{B}}^{0}$ and $\omega_{2} \in \Omega_{\mathrm{B}}^{2}$. Then

$$
\begin{aligned}
\mathfrak{t r}_{\Gamma}\left(\omega_{0} \omega_{2}\right) & =\operatorname{Tr}_{\text {Dix }}\left(\Gamma \omega_{0} \omega_{2}\right)=\operatorname{Tr}_{\text {Dix }}\left(\Gamma^{2} \omega_{2} \Gamma \omega_{0}\right) \\
& =\operatorname{Tr}_{\text {Dix }}\left(\Gamma \omega_{2} \omega_{0}\right)=\mathfrak{t r}_{\Gamma}\left(\omega_{2} \omega_{0}\right)
\end{aligned}
$$

where the second equality follows in view of the cyclicity of the Dixmier trace and the identity $\Gamma^{2}=\mathbf{1}$, while the second follows from $\Gamma \omega_{2} \Gamma=\omega_{2}+Z$ for some $Z \in \mathfrak{S}^{1}$ in view of Proposition 2.2 (3). The second case consists in $\omega, \omega^{\prime} \in \Omega_{\mathrm{B}}^{1}$. By linearity it is enough to prove the claim for elements of the type $\omega=\eta_{0} d_{B} \eta_{1}$, and $\omega^{\prime}=\eta_{0}^{\prime} d_{B} \eta_{1}^{\prime}$ with $\eta_{j}, \eta_{j}^{\prime} \in \pi\left(\mathscr{B}_{B}\right)^{+}$and $j=0,1$. We can use the same strategy of the computation (2.11). The first step consists in justifying the equality

$$
\operatorname{Tr}_{\text {Dix }}\left(\Gamma \omega \omega^{\prime}\right)=\operatorname{Tr}_{\text {Dix }}\left(\omega^{\prime} \Gamma \omega\right) \text {. }
$$

This is true since $\Gamma \omega \omega^{\prime}$ and $\omega^{\prime} \Gamma \omega$ are both in the Dixmier ideal and have the same system of non-zero eigenvalues [BS, Section 3.10, Theorem 5]. Therefore, the results follows in view of the Lidskii's formula for the Dixmier trace [LSZ, Theorem 7.3.1]. The second step consists in proving that $\Gamma \omega^{\prime} \Gamma=-\omega^{\prime}+R$ with a remainder $R$ such that $R \omega \in \mathfrak{S}^{1}$. This follows again from Proposition 2.2 (3). Summing up one gets

$$
\mathfrak{t r}_{\Gamma}\left(\omega \omega^{\prime}\right)=\operatorname{Tr}_{\text {Dix }}\left(\Gamma \omega \omega^{\prime}\right)=-\operatorname{Tr}_{\text {Dix }}\left(\Gamma \omega^{\prime} \omega\right)=-\mathfrak{t r}_{\Gamma}\left(\omega^{\prime} \omega\right)
$$

and the proof is completed.

As a consequence of Proposition 2.9 one has that the triple $\left(\Omega_{\mathrm{B}}^{\bullet}, \mathrm{d}_{\mathrm{B}}, \mathfrak{t r}_{\Gamma}\right)$ provides a quasi-cycle of dimension 2 over the algebra $\mathscr{S}_{\mathrm{B}}$ in the sense of Definition 2.7. We will refer to $\left(\Omega_{\mathrm{B}}^{\bullet}, \mathrm{d}_{\mathrm{B}}, \mathfrak{t r}_{\Gamma}\right)$ as the magnetic quasi-cycle.

2.4. Chern character. The following definition is adapted from [Con, Chapet 3 , Section 1. $\alpha$ ] or [GVF, Definition 8.17].

Definition 2.10 (Character of a quasi-cycle). Let $(\mathcal{H}, \mathrm{F})$ be a Fredholm module over $\mathscr{A}$ with a quasi-even structure of dimension $k$ with respect to $(\Gamma, \mathfrak{Z})$. Let $\left(\Omega \bullet, d, \mathfrak{t r}_{\Gamma}\right)$ be the associated quasi-cycle (of dimension k) over $\mathscr{A}$ according to Definition 2.7. The 
character of the quasi-cycle is the $(\mathrm{k}+1)$-linear functional $\tau_{\mathrm{k}}: \mathscr{A}^{\mathrm{k}+1} \rightarrow \mathbb{C}$ defined by

$$
\tau_{k}\left(A_{0}, A_{1}, \ldots, A_{k}\right):=\mathfrak{t r}_{\Gamma}\left(\pi\left(A_{0}\right) \mathrm{d} \pi\left(A_{1}\right), \ldots, \mathrm{d} \pi\left(A_{k}\right)\right)
$$

As discussed in Remark 2.8, after the passage to the quotient a quasi-cycle (of dimension $k$ ) defines a genuine cycle (of dimension $k$ ). Therefore, one can repeat verbatim the proof of [Con, Chapter 3, Section 1. $\alpha$, Proposition 4] or [GVF, Proposition 8.12] to deduce the following result:

Proposition 2.11. The character $\tau_{\mathrm{k}}$ of the quasi-cycle $\left(\Omega^{\bullet}, \mathrm{d}, \mathfrak{t r}_{\Gamma}\right)$ is a cyclic $k$-cocycle of the algebra $\mathscr{A}$.

In the case of interest of the magnetic quasi-cycle $\left(\Omega_{\mathrm{B}}^{\bullet}, \mathrm{d}_{\mathrm{B}}, \mathfrak{t r}_{\Gamma}\right)$ the associate character is given (up to the multiplicative prefactor $1 / 2$ ) by the trilinear map $\tau_{\mathrm{B}, 2}$ defined by (1.41). Therefore one has

Corollary 2.12. The trilinear functional $\tau_{\mathrm{B}, 2}$ defined by (1.41) is a cyclic 2-cocycle of the algebra $\mathscr{S}_{\mathrm{B}}$.

Following [Con, Section IV.1.ß] we will refer to the class of $\tau_{\mathrm{B}, 2}$ in $\mathrm{HC}^{\operatorname{even}}\left(\mathscr{S}_{\mathrm{B}}\right)$ as the Chern character of the quasi-even Fredholm module $\left(\mathcal{H}_{4}, \mathrm{~F}_{\mathrm{B}, \varepsilon}\right)$, and we will denote it with $\mathrm{Ch}_{2}\left(\mathcal{H}_{4}, \mathrm{~F}_{\mathrm{B}, \varepsilon}\right):=\left[\tau_{\mathrm{B}, 2}\right] \in \operatorname{HC}^{\text {even }}\left(\mathscr{S}_{\mathrm{B}}\right)$.

Consider the trilinear functional $\mathfrak{C h}_{\mathrm{B}}$ on $\mathscr{S}_{\mathrm{B}}$ defined by (1.32). As a consequence of Lemma 1.2 and Lemma 1.5 one obtains the equality

$$
\mathfrak{C h}_{\mathrm{B}}\left(\mathrm{A}_{0}, \mathrm{~A}_{1}, \mathrm{~A}_{2}\right)=\tau_{\mathrm{B}, 2}\left(\mathrm{~A}_{0}, \mathrm{~A}_{1}, \mathrm{~A}_{2}\right), \quad \forall \mathrm{A}_{0}, \mathrm{~A}_{1}, \mathrm{~A}_{2} \in \mathscr{S}_{\mathrm{B}}
$$

The latter equality represents a stronger version of [Con, Section IV.2. $\gamma$, Theorem 8] or [GVF, Theorem 10.32] and provides an incarnation of the celebrated local index formula of Connes and Moscovici [CM].

\section{Direct PROOF OF THE SECOND CONNES' FORMUla}

In this section we will provide the proof of the two versions of the Second Connes' formula anticipated in Section 1.2.

Proof of Lemma 1.2. The commutator $\left[D_{B}, \pi(A)\right]$ is well-defined for every $A \in \mathscr{S}_{\mathrm{B}}[\mathrm{DS}$, Proposition 3.2], and in view of (A.11) one gets

$$
\begin{aligned}
{\left[\mathrm{D}_{\mathrm{B}}, \pi(\mathrm{A})\right] } & =\left[\mathrm{D}_{\mathrm{B},-}, \pi(\mathrm{A})\right] \\
& =\left[\mathrm{K}_{1}, \mathrm{~A}\right] \otimes \frac{\gamma_{1}}{\sqrt{2}}+\left[\mathrm{K}_{2}, \mathrm{~A}\right] \otimes \frac{\gamma_{2}}{\sqrt{2}} \\
& =\nabla_{1} A \otimes \frac{\mathrm{i} \gamma_{2}}{\sqrt{2} \ell_{\mathrm{B}}}-\nabla_{2} A \otimes \frac{\mathrm{i} \gamma_{1}}{\sqrt{2} \ell_{B}},
\end{aligned}
$$


where $K_{1}$ and $K_{2}$ are the magnetic momenta (1.7), and $\nabla_{1}$ and $\nabla_{2}$ are the spatial derivations (1.13). Therefore,

$$
\begin{aligned}
{\left[D_{B}, \pi\left(A_{1}\right)\right]\left[D_{B}, \pi\left(A_{2}\right)\right]=} & -\frac{1}{2 \ell_{B}^{2}}\left(\nabla_{1} A_{1} \nabla_{1} A_{2} \otimes \gamma_{2}^{2}+\nabla_{2} A_{1} \nabla_{2} A_{2} \otimes \gamma_{1}^{2}\right) \\
& +\frac{1}{2 \ell_{B}^{2}}\left(\nabla_{1} A_{1} \nabla_{2} A_{2} \otimes \gamma_{2} \gamma_{1}+\nabla_{2} A_{1} \nabla_{1} A_{2} \otimes \gamma_{1} \gamma_{2}\right),
\end{aligned}
$$

for every $\mathrm{A}_{1}, \mathrm{~A}_{2} \in \mathscr{S}_{\mathrm{B}}$. Let us introduce the notation

$$
\begin{aligned}
& \delta_{0}\left(A_{1}, A_{2}\right):=\nabla_{1} A_{1} \nabla_{1} A_{2}+\nabla_{2} A_{1} \nabla_{2} A_{2}, \\
& \delta_{1}\left(A_{1}, A_{2}\right):=\nabla_{1} A_{1} \nabla_{2} A_{2}-\nabla_{2} A_{1} \nabla_{1} A_{2} .
\end{aligned}
$$

Since the algebra $\mathscr{S}_{\mathrm{B}}$ is closed under the action of the derivations, one has that both $\delta_{0}\left(A_{1}, A_{2}\right)$ and $\delta_{1}\left(A_{1}, A_{2}\right)$ are in $\mathscr{S}_{\mathrm{B}}$. By observing that $\gamma_{j}^{2}=\mathbf{1}_{4}$ is the $4 \times 4$ identity matrix for every $j=1,2,3,4$, one gets that

$$
\left[D_{B}, \pi\left(A_{1}\right)\right]\left[D_{B}, \pi\left(A_{2}\right)\right]=-\frac{1}{2 \ell_{B}^{2}} \pi\left(\delta_{0}\left(A_{1}, A_{2}\right)\right)+\frac{i}{2 \ell_{B}^{2}} \pi\left(\delta_{1}\left(A_{1}, A_{2}\right)\right) \Gamma
$$

where $\Gamma$ is the involution defined by (1.31). As a consequence, it follows that

$$
\begin{aligned}
\Gamma \pi\left(A_{0}\right)\left[D_{B}, \pi\left(A_{1}\right)\right]\left[D_{B}, \pi\left(A_{2}\right)\right]= & -\frac{1}{2 \ell_{B}^{2}} \pi\left(A_{0} \delta_{0}\left(A_{1}, A_{2}\right)\right) \Gamma \\
& +\frac{i}{2 \ell_{B}^{2}} \pi\left(A_{0} \delta_{1}\left(A_{1}, A_{2}\right)\right) .
\end{aligned}
$$

In view of (1.27), one has that

$$
\left|D_{B, \varepsilon}\right|^{-2} \Gamma \pi\left(A_{0}\right)\left[D_{B}, \pi\left(A_{1}\right)\right]\left[D_{B}, \pi\left(A_{2}\right)\right] \in \mathfrak{S}_{m}^{1^{+}},
$$

for every $A_{0}, A_{1}, A_{2} \in \mathscr{S}_{\mathrm{B}}$. Therefore, one is allowed to compute the Dixmier trace, and by linearity one gets that

$$
\mathfrak{C h}_{B}\left(A_{0}, A_{1}, A_{2}\right)=\frac{i}{4 \ell_{B}^{2}}\left(F_{1}\left(A_{0}, A_{1}, A_{2}\right)+i F_{0}\left(A_{0}, A_{1}, A_{2}\right)\right),
$$

where

$$
\begin{aligned}
& F_{0}\left(A_{0}, A_{1}, A_{2}\right):=\operatorname{Tr}_{\text {Dix }}\left(\left|D_{B, \varepsilon}\right|^{-2} \pi\left(A_{0} \delta_{0}\left(A_{1}, A_{2}\right)\right) \Gamma\right) \\
& F_{1}\left(A_{0}, A_{1}, A_{2}\right):=\operatorname{Tr}_{\text {Dix }}\left(\left|D_{B, \varepsilon}\right|^{-2} \pi\left(A_{0} \delta_{1}\left(A_{1}, A_{2}\right)\right)\right) .
\end{aligned}
$$

By using [DGM, Lemma B.3] and the diagonal representation of $\left|D_{B, \varepsilon}\right|^{-2}=\left(D_{B}^{2}+\varepsilon \mathbf{I}\right)^{-1}$ in terms of the harmonic oscillator $\mathrm{Q}_{\mathrm{B}}$, one gets that

$$
F_{0}\left(A_{0}, A_{1}, A_{2}\right)=\operatorname{Tr}_{\text {Dix }}\left(\frac{1}{Q_{B}+\xi \mathbf{I}} A_{0} \delta_{0}\left(A_{1}, A_{2}\right)\right) \operatorname{Tr}_{\mathbb{C}^{4}}\left(\mathrm{i} \gamma_{1} \gamma_{2}\right)=0,
$$

since $\operatorname{Tr}_{\mathbb{C}^{4}}\left(\mathrm{i} \gamma_{1} \gamma_{2}\right)=0$. It is worth remarking that the equality is justified by the fact that the first factor of the central term is well-defined for every $\xi>-1$ and its value does not 
depend on $\xi$ [DS, Proposition 2.27]. With a similar argument one gets

$$
F_{1}\left(A_{0}, A_{1}, A_{2}\right)=4 \operatorname{Tr}_{\text {Dix }}\left(\frac{1}{Q_{B}+\xi \mathbf{I}} A_{0} \delta_{1}\left(A_{1}, A_{2}\right)\right)
$$

where the pre-factor comes from $\operatorname{Tr}_{\mathbb{C}^{4}}\left(\mathbf{1}_{4}\right)=4$. By putting together all these results, one gets

$$
\begin{aligned}
\mathfrak{C h}_{\mathrm{B}}\left(A_{0}, A_{1}, A_{2}\right) & =\frac{\mathrm{i}}{\ell_{\mathrm{B}}^{2}} \operatorname{Tr}_{\text {Dix }}\left(\frac{1}{\mathrm{Q}_{\mathrm{B}}+\xi_{\mathbf{1}}} A_{0} \delta_{1}\left(A_{1}, A_{2}\right)\right) \\
& =\frac{\mathrm{i}}{\ell_{\mathrm{B}}^{2}} f_{\mathrm{B}}\left(A_{0} \delta_{1}\left(A_{1}, A_{2}\right)\right) \\
& =\frac{\mathrm{i}}{\ell_{\mathrm{B}}^{2}} \Psi_{\mathrm{B}}\left(A_{0}, A_{1}, A_{2}\right),
\end{aligned}
$$

where the second equality is proved in [DS, Proposition 2.27] and the third equality follows from (1.15). This concludes the proof.

Remark 3.1. The same proof described above can be adapted to prove the claim of Remark 1.4 about the triviality of $\widehat{\mathfrak{C h}_{\mathrm{B}}}$. The main difference relies in the equality

$$
\begin{aligned}
\chi \pi\left(A_{0}\right)\left[D_{B}, \pi\left(A_{1}\right)\right]\left[D_{B}, \pi\left(A_{2}\right)\right]= & -\frac{1}{2 \ell_{B}^{2}} \pi\left(A_{0} \delta_{0}\left(A_{1}, A_{2}\right)\right) \chi \\
& +\frac{i}{2 \ell_{B}^{2}} \pi\left(A_{0} \delta_{1}\left(A_{1}, A_{2}\right)\right) \chi \Gamma .
\end{aligned}
$$

In the computation of the Dixmier trace both summands produce vanishing terms. The first summand vanishes since $\chi$ is responsible for a term proportional to $\operatorname{Tr}_{\mathbb{C}^{4}}\left(\gamma_{1} \gamma_{2} \gamma_{3} \gamma_{4}\right)=$ 0 and the second summand vanishes since $\chi \Gamma$ is responsible for a term proportional to $\operatorname{Tr}_{\mathbb{C}^{4}}\left(\gamma_{3} \gamma_{4}\right)=0$

As a preparation for the proof of Lemma 1.5 let us anticipate a result which improves [DS, Lemma 3.14]. For that we need to define the expression

$$
\widetilde{\mathrm{I}_{0}}\left(A_{1}, A_{2}\right):=\left|D_{\mathrm{B}, \varepsilon}\right|^{-2}\left[\mathrm{D}_{\mathrm{B}}, \pi\left(A_{1}\right)\right]\left[\mathrm{D}_{\mathrm{B}}, \pi\left(A_{2}\right)\right], \quad A_{1}, A_{2} \in \mathscr{S}_{\mathrm{B}} .
$$

Lemma 3.2. Let $\mathrm{A}_{1}, \mathrm{~A}_{2} \in \mathscr{S}_{\mathrm{B}}$. Then it holds true that

$$
\left[F_{B, \varepsilon}, \pi\left(A_{1}\right)\right]\left[F_{B, \varepsilon}, \pi\left(A_{2}\right)\right]=\tilde{I_{0}}\left(A_{1}, A_{2}\right)+Z\left(A_{1}, A_{2}\right)
$$

with $\mathrm{Z}\left(\mathrm{A}_{1}, \mathrm{~A}_{2}\right) \in \mathfrak{S}^{1}$.

Proof. The starting point of the proof is decomposition of the product

$$
\left[F_{B, \varepsilon}, \pi\left(A_{1}\right)\right]\left[F_{B, \varepsilon}, \pi\left(A_{2}\right)\right]:=\sum_{i=0}^{3} I_{i}\left(A_{1}, A_{2}\right)
$$


as presented in [DS, eq. (3.12)]. By using the equalities $\left[\mathrm{F}_{\mathrm{B}, \varepsilon}, \mathrm{T}^{*}\right]^{*}=-\left[\mathrm{F}_{\mathrm{B}, \varepsilon}, \mathrm{T}\right]$ and $\left[\mathrm{D}_{\mathrm{B}}, \mathrm{T}^{*}\right]^{*}=-\left[\mathrm{D}_{\mathrm{B}}, \mathrm{T}\right]$ for every $\mathrm{T} \in \pi\left(\mathscr{S}_{\mathrm{B}}\right)$ one gets

$$
\begin{aligned}
& \mathrm{I}_{0}\left(A_{1}, A_{2}\right):=\left|\mathrm{D}_{\mathrm{B}, \varepsilon}\right|^{-1}\left[\mathrm{D}_{\mathrm{B}}, \pi\left(\mathrm{A}_{1}\right)\right]\left[\mathrm{D}_{\mathrm{B}}, \pi\left(A_{2}\right)\right]\left|\mathrm{D}_{\mathrm{B}, \varepsilon}\right|^{-1} \\
& \mathrm{I}_{1}\left(A_{1}, A_{2}\right):=\left|\mathrm{D}_{\mathrm{B}, \varepsilon}\right|^{-1}\left[\mathrm{D}_{\mathrm{B}}, \pi\left(A_{1}\right)\right] \mathrm{D}_{\mathrm{B}}\left[\left|\mathrm{D}_{\mathrm{B}, \varepsilon}\right|^{-1}, \pi\left(A_{2}\right)\right] \\
& \mathrm{I}_{2}\left(A_{1}, A_{2}\right):=\left[\left|\mathrm{D}_{\mathrm{B}, \varepsilon}\right|^{-1}, \pi\left(A_{1}\right)\right] \mathrm{D}_{\mathrm{B}}\left[\mathrm{D}_{\mathrm{B}}, \pi\left(A_{2}\right)\right]\left|\mathrm{D}_{\mathrm{B}, \varepsilon}\right|^{-1} \\
& \mathrm{I}_{3}\left(A_{1}, A_{2}\right):=\left[\left|\mathrm{D}_{\mathrm{B}, \varepsilon}\right|^{-1}, \pi\left(A_{1}\right)\right] \mathrm{D}_{\mathrm{B}}^{2}\left[\left|\mathrm{D}_{\mathrm{B}, \varepsilon}\right|^{-1}, \pi\left(A_{2}\right)\right] .
\end{aligned}
$$

Let us observe that a direct computation shows that $\left[\left|\mathrm{D}_{B, \varepsilon}\right|^{-1}, \pi(A)\right]$ is a diagonal matrix with entries given by $C_{\varepsilon+s, \varepsilon+s}(A), s \in\{0, \pm 1\}$, where the notation of Lemma A.6 has been used. As a consequence it turns out that $\left[\left|\mathrm{D}_{\mathrm{B}, \varepsilon}\right|^{-1}, \pi(A)\right] \in \mathfrak{S}^{1}$ for every $A \in \mathscr{S}_{\mathrm{B}}$. Let us focus on the term $I_{1}\left(A_{1}, A_{2}\right)=B S$, where $S:=F_{B, \varepsilon}\left[\left|D_{B, \varepsilon}\right|^{-1}, \pi\left(A_{2}\right)\right]$ is a trace-class operator and

$$
\begin{aligned}
\mathrm{B} & :=\left|\mathrm{D}_{\mathrm{B}, \varepsilon}\right|^{-1}\left[\mathrm{D}_{\mathrm{B}}, \pi\left(A_{1}\right)\right]\left|\mathrm{D}_{\mathrm{B}, \varepsilon}\right| \\
& =-\left|\mathrm{D}_{\mathrm{B}, \varepsilon}\right|^{-1}\left[\left|\mathrm{D}_{\mathrm{B}, \varepsilon}\right|,\left[\mathrm{D}_{\mathrm{B}}, \pi\left(A_{1}\right)\right]\right]+\left[\mathrm{D}_{\mathrm{B}}, \pi\left(A_{1}\right)\right]
\end{aligned}
$$

is a bounded operator in view of [DS, Corollary 3.3] which shows that $\left[D_{B}, \pi\left(A_{1}\right)\right] \in$ $\mathscr{S}_{\mathrm{B}} \otimes \mathrm{Mat}_{4}(\mathbb{C})$, and [DS, Proposition 3.4] which shows that the commutator $\left[\left|\mathrm{D}_{\mathrm{B}, \varepsilon}\right|, \mathrm{T}\right]$ is bounded for every $T \in \mathscr{S}_{\mathrm{B}} \otimes \operatorname{Mat}_{4}(\mathbb{C})$. It follows that $\mathrm{I}_{1}\left(A_{1}, A_{2}\right) \in \mathfrak{S}^{1}$. From the identity $I_{2}\left(A_{1}, A_{2}\right)=I_{2}\left(A_{2}^{*}, A_{1}^{*}\right)^{*}$ one immediately concludes that $I_{2}\left(A_{1}, A_{2}\right) \in \mathfrak{S}^{1}$. Similarly, the term $I_{3}\left(A_{1}, A_{2}\right)=S^{\prime} B^{\prime}$ is the product of the trace-class operator $S^{\prime}:=$ $\left[\left|D_{B, \varepsilon}\right|^{-1}, \pi\left(A_{1}\right)\right]$ and the bounded operator

$$
\begin{aligned}
\mathrm{B}^{\prime}: & =\mathrm{D}_{\mathrm{B}}^{2}\left[\left|\mathrm{D}_{\mathrm{B}, \varepsilon}\right|^{-1}, \pi\left(A_{2}\right)\right] \\
& =\left[\mathrm{D}_{\mathrm{B}}^{2}\left|\mathrm{D}_{\mathrm{B}, \varepsilon}\right|^{-1}, \pi\left(A_{2}\right)\right]-\left[\mathrm{D}_{\mathrm{B}}^{2}, \pi\left(A_{2}\right)\right]\left|\mathrm{D}_{\mathrm{B}, \varepsilon}\right|^{-1} \\
& =\left[\left|\mathrm{D}_{\mathrm{B}, \varepsilon}\right|, \pi\left(\mathrm{A}_{2}\right)\right]-\varepsilon\left[\left|\mathrm{D}_{\mathrm{B}, \varepsilon}\right|^{-1}, \pi\left(\mathrm{A}_{2}\right)\right]-\left[\mathrm{D}_{\mathrm{B}}^{2}, \pi\left(\mathrm{A}_{2}\right)\right]\left|\mathrm{D}_{\mathrm{B}, \varepsilon}\right|^{-1}
\end{aligned}
$$

where in the last equality it has been used the identity $D_{B}^{2}=\left|D_{B, \varepsilon}\right|^{2}-\varepsilon \mathbf{I}$ and the boundedness of $\left[D_{B}^{2}, \pi\left(A_{2}\right)\right]$ is discussed in the proof of [DS, Lemma 3.9]. Therefore, one obtains that

$$
\begin{aligned}
{\left[F_{B, \varepsilon}, \pi\left(A_{2}\right)\right] } & =I_{0}\left(A_{1}, A_{2}\right)+Z^{\prime}\left(A_{1}, A_{2}\right) \\
& =\widetilde{I_{0}}\left(A_{1}, A_{2}\right)+Z^{\prime}\left(A_{1}, A_{2}\right)+Z^{\prime \prime}\left(A_{1}, A_{2}\right)
\end{aligned}
$$

with $Z^{\prime}\left(A_{1}, A_{2}\right)=\sum_{i=1}^{3} I_{i}\left(A_{1}, A_{2}\right) \in \mathfrak{S}^{1}$ and

$$
\begin{aligned}
Z^{\prime \prime}\left(A_{1}, A_{2}\right): & =I_{0}\left(A_{1}, A_{2}\right)-\widetilde{I_{0}}\left(A_{1}, A_{2}\right) \\
& =-\left|D_{B, \varepsilon}\right|^{-1}\left[\left|D_{B, \varepsilon}\right|^{-1},\left[D_{B}, \pi\left(A_{1}\right)\right]\left[D_{B}, \pi\left(A_{2}\right)\right]\right] .
\end{aligned}
$$

since $\left[D_{B}, \pi\left(A_{1}\right)\right]\left[D_{B}, \pi\left(A_{2}\right)\right]$ is a diagonal matrix with entries in $\mathscr{S}_{B}$ in view of (3.1) one gets that the commutator with $\left|D_{B, \varepsilon}\right|^{-1}$ diagonal matrix with entries of the type $C_{\varepsilon, \varepsilon}(A)$ with the notation of Lemma A.6. As a consequence $Z^{\prime \prime}\left(A_{1}, A_{2}\right) \in \mathfrak{S}^{1}$ and the claim is proved. 
Proof of Lemma 1.5. Since $\mathfrak{S}^{1} \subset \mathfrak{S}_{0}^{1^{+}}$, and the ideal $\mathfrak{S}_{0}^{1^{+}}$lies in the common kernel of all the Dixmier traces, one infers from Lemma 3.2 that

$$
\operatorname{Tr}_{\text {Dix }, \omega}\left(\mathrm{Yd}_{\mathrm{B}} \pi\left(A_{1}\right) \mathrm{d}_{\mathrm{B}} \pi\left(A_{2}\right)\right)=\operatorname{Tr}_{\text {Dix }, \omega}\left(\mathrm{Y} \widetilde{\mathrm{I}_{0}}\left(A_{1}, A_{2}\right)\right) .
$$

To prove Lemma 1.5 we have to fix $Y=\Gamma \pi\left(A_{0}\right)$ for some $A_{0} \in \mathscr{S}_{\mathrm{B}}$. Since $\Gamma$ commutes with $\left|\mathrm{D}_{\mathrm{B}, \varepsilon}\right|^{-2}$ and

$$
\pi\left(A_{0}\right)\left|D_{B, \varepsilon}\right|^{-2}-\left|D_{B, \varepsilon}\right|^{-2} \pi\left(A_{0}\right) \in \mathfrak{S}_{0}^{1^{+}}
$$

in view of Lemma A.2, one finally gets

$$
\begin{aligned}
\operatorname{Tr}_{\text {Dix }, \omega} & \left(\Gamma \pi\left(A_{0}\right) d_{B} \pi\left(A_{1}\right) d_{B} \pi\left(A_{2}\right)\right)= \\
& =\operatorname{Tr}_{\text {Dix }, \omega}\left(\left|D_{B, \varepsilon}\right|^{-2} \Gamma \pi\left(A_{0}\right)\left[D_{B}, \pi\left(A_{1}\right)\right]\left[D_{B}, \pi\left(A_{2}\right)\right]\right) .
\end{aligned}
$$

In view of (3.2) the operator inside the Dixmier trace on the right-hand side of the latter equation is a measurable element of the Dixmier ideal. As a consequence we do not have to specify a generalized limit for the computation of the Dixmier trace. In addition a comparison with (1.32) provides

$$
\operatorname{Tr}_{\text {Dix }}\left(\Gamma \pi\left(A_{0}\right) d_{B} \pi\left(A_{1}\right) d_{B} \pi\left(A_{2}\right)\right)=2 \mathfrak{C h}_{B}\left(A_{0}, A_{1}, A_{2}\right) .
$$

By using the notation of the compatible graded trace (1.40) and Lemma 1.2 one gets

$$
\mathfrak{t r}_{\Gamma}\left(\pi\left(A_{0}\right) d_{B} \pi\left(A_{1}\right) d_{B} \pi\left(A_{2}\right)\right)=\frac{i 2}{\ell_{B}^{2}} \Psi_{B}\left(A_{0}, A_{1}, A_{2}\right) .
$$

A comparison with definition (1.41) concludes the proof.

\section{Corollary 3.3. It holds true that}

$$
\mathfrak{t r}_{\Gamma}\left(\left[\mathrm{F}_{\mathrm{B}, \varepsilon}, \pi\left(A_{1}\right)\right]\left[\mathrm{F}_{\mathrm{B}, \varepsilon}, \pi\left(A_{2}\right)\right]\right)=0
$$

for every $A_{1}, A_{2} \in \mathscr{S}_{\mathrm{B}}$.

Proof. From Lemma 3.2 and the definition of the compatible graded trace (1.40) one obtains that

$$
\mathfrak{t r}_{\Gamma}\left(\left[\mathrm{F}_{\mathrm{B}, \varepsilon}, \pi\left(A_{1}\right)\right]\left[\mathrm{F}_{\mathrm{B}, \varepsilon}, \pi\left(A_{2}\right)\right]\right)=\operatorname{Tr}_{\text {Dix }}\left(\Gamma \widetilde{\mathrm{I}}_{0}\left(A_{1}, A_{2}\right)\right) .
$$

In view of equation (3.1) one gets

$$
\Gamma \widetilde{\mathrm{I}_{0}}\left(A_{1}, A_{2}\right)=-\frac{1}{2 \ell_{\mathrm{B}}^{2}}\left|\mathrm{D}_{\mathrm{B}, \varepsilon}\right|^{-2} \pi\left(\delta_{0}\left(A_{1}, A_{2}\right)\right) \Gamma+\frac{\mathrm{i}}{2 \ell_{\mathrm{B}}^{2}}\left|\mathrm{D}_{\mathrm{B}, \varepsilon}\right|^{-2} \pi\left(\delta_{1}\left(A_{1}, A_{2}\right) .\right)
$$


By using the vanishing of the trace of $\Gamma$ as in the proof of Lemma 1.2 one obtains

$$
\begin{aligned}
\mathfrak{t r}_{\Gamma}\left(\left[\mathrm{F}_{\mathrm{B}, \varepsilon}, \pi\left(A_{1}\right)\right]\left[\mathrm{F}_{\mathrm{B}, \varepsilon}, \pi\left(A_{2}\right)\right]\right) & =\frac{\mathrm{i}}{2 \ell_{\mathrm{B}}^{2}} \operatorname{Tr}_{\text {Dix }}\left(\left|\mathrm{D}_{\mathrm{B}, \varepsilon}\right|^{-2} \pi\left(\delta_{1}\left(A_{1}, A_{2}\right)\right)\right. \\
& =\frac{\mathrm{i} 2}{\ell_{\mathrm{B}}^{2}} \operatorname{Tr}_{\text {Dix }}\left(\frac{1}{\mathrm{Q}_{\mathrm{B}}+\xi \mathbf{I}} \delta_{1}\left(A_{1}, A_{2}\right)\right) \\
& =\frac{\mathrm{i} 2}{\ell_{\mathrm{B}}^{2}} f_{\mathrm{B}}\left(\delta_{1}\left(A_{1}, A_{2}\right)\right)
\end{aligned}
$$

where $\xi>-1$ and the last equality is a consequence of [DS, Proposition 2.27]. By using the Leibniz's rule for derivations $\nabla_{1}$ and $\nabla_{2}$ one obtains that

$$
\begin{aligned}
& \nabla_{1} A_{1} \nabla_{2} A_{2}=\nabla_{1}\left(A_{1} \nabla_{2} A_{2}\right)-A_{1}\left(\nabla_{1} \circ \nabla_{2} A_{2}\right), \\
& \nabla_{2} A_{1} \nabla_{1} A_{2}=\nabla_{2}\left(A_{1} \nabla_{1} A_{2}\right)-A_{1}\left(\nabla_{2} \circ \nabla_{1} A_{2}\right) .
\end{aligned}
$$

Since the derivations $\nabla_{1}$ and $\nabla_{2}$ commute one gets

$$
\delta_{1}\left(A_{1}, A_{2}\right):=\nabla_{1}\left(A_{1} \nabla_{2} A_{2}\right)-\nabla_{2}\left(A_{1} \nabla_{1} A_{2}\right) .
$$

The property $f_{\mathrm{B}} \circ \nabla_{j}=0$ for $j=1,2$ (cf. [DS, Section 2.8]) concludes the proof.

\section{APPEndiX A. TEChNiCALities}

A.1. Weak $L^{p}$-spaces. The information contained in this section is quite standard and can be found in numerous publications existing in the literature. For the benefit of the reader we will refer mainly to [Pie, Sim1, Sim2, Con, LSZ, AMSZ].

Let $\mathrm{T} \in \mathscr{K}(\mathcal{H})$ be a compact operator. The Schatten quasi-norm of order $p>0$ is defined as

$$
\|\mathrm{T}\|_{p}:=\left(\sum_{m \in \mathbb{N}_{0}} \mu_{m}(\mathrm{~T})^{p}\right)^{\frac{1}{p}}
$$

where $\mu_{m}(T)$ denotes the sequence of singular values of $T$ listed in decreasing order and repeated according to their multiplicity. This is a norm for $p \geqslant 1$. The corresponding Schatten ideal of order $p$ is defined as

$$
\mathfrak{S}^{p}:=\left\{\mathrm{T} \in \mathscr{K}(\mathcal{H}) \mid\|\mathrm{T}\|_{p}<+\infty\right\} .
$$

The weak Schatten quasi-norm of order $p>0$ is defined as

$$
\|\mathrm{T}\|_{\mathrm{p}^{+}}^{\star}:=\sup _{\mathrm{m} \geqslant 0}\left\{(\mathrm{~m}+1)^{\frac{1}{p}} \mu_{m}(\mathrm{~T})\right\}
$$

The weak Schatten ideal of order $p$ is given by

$$
\mathfrak{S}_{\mathrm{w}}^{\mathrm{p}}:=\left\{\mathrm{T} \in \mathscr{K}(\mathcal{H}) \mid\|\mathrm{T}\|_{\mathrm{p}^{+}}^{\star}<+\infty\right\} .
$$

This is a two sided quasi-Banach ideal. The minimal ideal $\mathfrak{S}_{\mathrm{w}, 0}^{\mathrm{p}} \subset \mathfrak{S}_{\mathrm{w}}^{\mathrm{p}}$ is defined by

$$
\mathfrak{S}_{\mathrm{w}, 0}^{\mathrm{p}}:=\left\{\begin{array}{l|l}
\mathrm{T} \in \mathfrak{S}_{\mathrm{w}}^{\mathrm{p}} & \lim _{\mathrm{m} \rightarrow+\infty}(\mathrm{m}+1)^{\frac{1}{\mathrm{p}}} \mu_{\mathrm{m}}(\mathrm{T})=0
\end{array}\right\} .
$$


The quasi-norm (A.2) meets the following algebraic properties

$$
\begin{aligned}
& \|A T\|_{\mathrm{p}^{+}}^{\star} \leqslant\|A\|\|\mathrm{T}\|_{\mathrm{p}^{+}}^{\star}, \quad\|\mathrm{TA}\|_{\mathrm{p}^{+}}^{\star} \leqslant\|A\|\|\mathrm{T}\|_{\mathrm{p}^{+}}^{\star}, \\
& \left\|\mathrm{T}_{1}+\mathrm{T}_{2}\right\|_{\mathrm{p}^{+}}^{\star} \leqslant 2^{\frac{1}{\mathrm{p}}}\left(\left\|\mathrm{T}_{1}\right\|_{\mathrm{p}^{+}}^{\star}+\left\|\mathrm{T}_{2}\right\|_{\mathrm{p}^{+}}^{\star}\right),
\end{aligned}
$$

valid for every $T, T_{1}, T_{2} \in \mathfrak{S}_{\mathrm{w}}^{\mathrm{p}}$ and $A \in \mathscr{B}(\mathcal{H})$. Moreover, one has a Hölder type inequality,

$$
\left\|T_{1} T_{2}\right\|_{r^{+}}^{\star} \leqslant 2^{\frac{1}{r}}\left\|T_{1}\right\|_{p^{+}}^{\star}\left\|T_{2}\right\|_{q^{+}}^{*}
$$

valid for every $T_{1} \in \mathfrak{S}_{\mathrm{w}}^{\mathrm{p}}$ and $\mathrm{T}_{2} \in \mathfrak{S}_{\mathrm{w}}^{\mathrm{q}}$ such that $\mathrm{r}^{-1}=\mathrm{p}^{-1}+\mathrm{q}^{-1}$. A proof of (A.3) and (A.4) can be found in [Sim1, Theorem 2.1]. For $p>q$, one can prove that [AMSZ, eq. (2.2)]

$$
\|T\|_{p} \leqslant \mathfrak{Z}\left(\frac{p}{q}\right)^{\frac{1}{p}}\|T\|_{q^{+}}^{\star}
$$

where \|\|$_{p}$ is the norm of the Schatten ideal $\mathfrak{S}^{p}$. As a consequence one obtains the continuous embeddings $\mathfrak{S}_{w}^{q} \subset \mathfrak{S}^{p}$ if $p>q$. Since the condition $T \in \mathfrak{S}^{p}$ implies that $\mu_{m}(T)^{p}$ must decrease faster than $\mathrm{m}^{-1}$ one obtains the chain of continuous embeddings

$$
\mathfrak{S}_{\mathrm{w}}^{\mathrm{q}} \subset \mathfrak{S}^{\mathrm{p}} \subset \mathfrak{S}_{\mathrm{w}, 0}^{\mathrm{p}} \subset \mathfrak{S}_{\mathrm{w}}^{\mathrm{p}}, \quad 0<\mathrm{q}<\mathrm{p}<+\infty .
$$

For $p>1$ the weak Schatten quasi-norm is equivalent to Calderón norm

$$
\|\mathrm{T}\|_{p^{+}}:=\sup _{N \geqslant 1}\left\{\frac{1}{N^{1-\frac{1}{p}}} \sum_{m=0}^{N-1} \mu_{m}(T)\right\}
$$

since one has the inequalities

$$
\|T\|_{p^{+}}^{\star} \leqslant\|T\|_{p^{+}} \leqslant \frac{p}{p-1}\|T\|_{p^{+}}^{\star} .
$$

Therefore, when $p>1$ the spaces $\mathfrak{S}_{\mathrm{w}}^{\mathrm{p}}$ are indeed two-sided Banach ideals. In particular one has that $\mathfrak{S}_{\mathrm{w}}^{\mathrm{p}}=\mathfrak{S}^{\mathrm{p}^{+}}$coincides with the $\mathrm{p}$-th Dixmier ideal as defined in [DGM, Appendix B.1].

For $p=1$ the appropriate Calderón norm is

$$
\|\mathrm{T}\|_{1^{+}}:=\sup _{\mathrm{N} \geqslant 2}\left\{\frac{1}{\log (\mathrm{N})} \sum_{\mathrm{m}=0}^{\mathrm{N}-1} \mu_{\mathrm{m}}(\mathrm{T})\right\}
$$

and the corresponding Dixmier ideal is

$$
\mathfrak{S}^{1^{+}}:=\left\{\mathrm{T} \in \mathscr{K}(\mathcal{H}) \mid\|\mathrm{T}\|_{1^{+}}<+\infty\right\} .
$$

Also in this case $\mathfrak{S}_{0}^{1+}$ denotes the closure of the finite-rank operators in the Calderón norm \|\|$_{1^{+}}$. In this case the weak Schatten ideal $\mathfrak{S}_{\mathrm{w}}^{1}$ is just smaller than $\mathfrak{S}^{1^{+}}$. In fact one has the chain of strict inclusions

$$
\mathfrak{S}^{1} \subset \mathfrak{S}_{0}^{1^{+}} \subset \mathfrak{S}_{\mathrm{w}}^{1} \subset \mathfrak{S}^{1^{+}}
$$


For $\mathrm{q} \geqslant 1$, let us introduce the Mačaev norm

$$
\|\mathrm{T}\|_{q^{-}}:=\sum_{m=0}^{+\infty} \frac{\mu_{m}(T)}{(m+1)^{1-\frac{1}{q}}}
$$

and the Mačaev ideal of order $q$

$$
\mathfrak{S}^{\mathrm{q}^{-}}:=\left\{\mathrm{T} \in \mathscr{K}(\mathcal{H}) \mid\|\mathrm{T}\|_{\mathrm{q}^{-}}<+\infty\right\} .
$$

one has the following strict inclusions

$$
\mathfrak{S}^{\mathrm{s}^{+}} \subset \mathfrak{S}^{\mathrm{q}^{-}} \subset \mathfrak{S}^{\mathrm{q}} \subset \mathfrak{S}^{\mathrm{q}^{+}}, \quad 1 \leqslant \mathrm{~s}<\mathrm{q}<+\infty .
$$

The space $\mathfrak{S}^{p^{+}}$is the dual of $\mathfrak{S}^{q^{-}}$when $q^{-1}+p^{-1}=1, p, q \geqslant 1$. This means that if $\mathrm{T} \in \mathfrak{S}^{\mathrm{p}^{+}}$and $\mathrm{S} \in \mathfrak{S}^{\mathrm{q}^{-}}$, then $\mathrm{TS} \in \mathfrak{S}^{1}$ and $\|\mathrm{TS}\|_{1} \leqslant\|\mathrm{~T}\|_{\mathrm{p}^{+}}\|\mathrm{S}\|_{\mathrm{q}^{-}}$.

A.2. Relevant trace-class elements. Let $\mathrm{Q}_{\mathrm{B}}$ be the harmonic oscillator (1.25), and let us define

$$
\mathrm{Q}_{\mathrm{B}, \varepsilon}:=\mathrm{Q}_{\mathrm{B}}+\varepsilon \mathbf{I}, \quad \varepsilon>-1 .
$$

Lemma A.1. It holds true that

$$
\mathrm{C}_{\varepsilon, \varepsilon^{\prime}}(\mathrm{A}):=\mathrm{Q}_{\mathrm{B}, \varepsilon}^{-\frac{1}{2}} \mathrm{~A}-\mathrm{A} \mathrm{Q}_{\mathrm{B}, \varepsilon^{\prime}}^{-\frac{1}{2}} \in \mathfrak{S}^{1}
$$

for every $\mathrm{A} \in \mathscr{S}_{\mathrm{B}}$, independently of $\varepsilon, \varepsilon^{\prime}>-1$.

Proof. Let us start with the simple case of $A=\Upsilon_{j \mapsto k}$, where the transition operator is defined by (1.3). In the proof of [DS, Lemma 3.14] it has been proved that

$$
\mathrm{C}_{\varepsilon, \varepsilon^{\prime}}\left(\Upsilon_{j \mapsto k}\right):=\mathrm{Q}_{\mathrm{B}, \varepsilon}^{-\frac{1}{2}} \Upsilon_{j \mapsto k}-\Upsilon_{j \mapsto k} \mathrm{Q}_{\mathrm{B}, \varepsilon^{\prime}}^{-\frac{1}{2}} \in \mathfrak{S}^{1} \subset \mathfrak{S}_{0}^{1^{+}}
$$

is a trace class element. This follows by observing that the singular values are given by

$$
\mu_{m}\left[C_{\varepsilon, \varepsilon^{\prime}}\left(\Upsilon_{j \mapsto k}\right)\right]:=\frac{\alpha_{m}^{(j, k)}}{(m+1)^{\frac{3}{2}}} \propto(m+1)^{-\frac{3}{2}}
$$

with

$$
\alpha_{m}^{(j, k)}:=\frac{\left|\zeta_{j}-\zeta_{k}\right|}{\sqrt{1+\frac{\zeta_{j}}{m+1}} \sqrt{1+\frac{\zeta_{k}}{m+1}}\left(\sqrt{1+\frac{\zeta_{j}}{m+1}}+\sqrt{1+\frac{\zeta_{k}}{m+1}}\right)} \leqslant \frac{\left|\zeta_{j}-\zeta_{k}\right|}{2}
$$

where $\zeta_{j}:=j+\varepsilon^{\prime}, \zeta_{k}:=k+\varepsilon$. Moreover, from the explicit form of the singular values one infers that

$$
\left\|C_{\varepsilon, \varepsilon^{\prime}}\left(\Upsilon_{j \mapsto k}\right)\right\|_{1} \leqslant \frac{1}{2} \mathfrak{Z}\left(\frac{3}{2}\right)\left|\zeta_{j}-\zeta_{k}\right|
$$

where $\mathfrak{Z}$ denotes the Riemann zeta function. Now, let us consider a generic element $A=\sum_{(j, k) \in \mathbb{N}_{0}^{2}} a_{j, k} \Upsilon_{j \mapsto k}$ with $\left\{a_{j, k}\right\} \in S\left(\mathbb{N}_{0}^{2}\right)$. The linearity of the commutator, the triangular inequality and the Cauchy-Schwarz inequality imply

$$
\left\|Q_{B, \varepsilon}^{-\frac{1}{2}} A-A Q_{B, \varepsilon}^{-\frac{1}{2}}\right\|_{1} \leqslant \frac{1}{2} \mathfrak{Z}\left(\frac{3}{2}\right) \sum_{(j, k) \in \mathbb{N}_{0}^{2}}\left|\zeta_{j}-\zeta_{k}\right|\left|a_{j, k}\right| \leqslant M_{\varepsilon, \varepsilon^{\prime}}^{p} r_{p}\left(\left\{a_{j, k}\right\}\right)
$$


where

$$
M_{\varepsilon, \varepsilon^{\prime}}^{p}:=\frac{1}{2} \mathfrak{Z}\left(\frac{3}{2}\right)\left(\sum_{(j, k) \in \mathbb{N}_{0}^{2}} \frac{\left|\zeta_{j}-\zeta_{k}\right|}{(2 j+1)^{p}(2 k+1)^{p}}\right)^{\frac{1}{2}}
$$

is a finite constant whenever $p>2$ and

$$
r_{p}\left(\left\{a_{j, k}\right\}\right):=\left(\sum_{(j, k) \in \mathbb{N}_{0}^{2}}(2 j+1)^{p}(2 k+1)^{p}\left|a_{j, k}\right|^{2}\right)^{\frac{1}{2}}
$$

is the $p$-th Schwarz semi-norm of the Fréchet space $S\left(\mathbb{N}_{0}^{2}\right)$. This proves that $C_{\varepsilon, \varepsilon^{\prime}}: \mathscr{S}_{\mathrm{B}} \rightarrow$ $\mathfrak{S}^{1}$ is a continuous map.

The next result can be proved along the same lines as those of the proof of Lemma A.6. However, it provides two vanishing criteria for elements in $\mathscr{L}_{\mathrm{B}}^{1}$ and not only in $\mathscr{S}_{\mathrm{B}}$. The proof of the next result justifies the formulas anticipated after [DS, Proposition 2.27] .

Lemma A.2. It holds true that

$$
\mathrm{D}_{\varepsilon, \varepsilon^{\prime}}(\mathrm{A}):=\mathrm{Q}_{\mathrm{B}, \varepsilon}^{-1} \mathrm{~A}-\mathrm{A} \mathrm{Q}_{\mathrm{B}, \varepsilon^{\prime}}^{-1} \in \mathfrak{S}_{0}^{1^{+}},
$$

and

$$
\mathrm{J}_{\varepsilon, \varepsilon^{\prime}, \varepsilon^{\prime \prime}}(\mathrm{A}):=\mathrm{Q}_{\mathrm{B}, \varepsilon}^{-\frac{1}{2}} \mathrm{~A} \mathrm{Q}_{\mathrm{B}, \varepsilon^{\prime}}^{-\frac{1}{2}}-\mathrm{Q}_{\mathrm{B}, \varepsilon^{\prime \prime}}^{-1} \mathrm{~A} \in \mathfrak{S}_{0}^{1^{+}},
$$

for every $\mathrm{A} \in \mathscr{L}_{\mathrm{B}}^{1}$, independently of $\varepsilon, \varepsilon^{\prime}, \varepsilon^{\prime \prime}>-1$.

Proof. Let us start with the simple case of $A=\Upsilon_{j \mapsto k}$. By using the same notation in [DS, Corollary 2.26] one gets the spectral resolution

$$
\mathrm{D}_{\varepsilon, \varepsilon^{\prime}}\left(\Upsilon_{j \mapsto k}\right)=\left(\sum_{m \in \mathbb{N}_{0}} \frac{\zeta_{j}-\zeta_{k}}{\left(m+1+\zeta_{j}\right)\left(m+1+\zeta_{k}\right)} P_{m}\right) \Upsilon_{j \mapsto k},
$$

where $\zeta_{j}:=j+\varepsilon^{\prime}, \zeta_{k}:=k+\varepsilon$ and the $P_{m}$ are the dual Landau projections defined in [DS, eq. (2.15)]. It follows that

$$
\left|D_{\varepsilon, \varepsilon^{\prime}}\left(\Upsilon_{j \mapsto k}\right)\right|=\left(\sum_{m \in \mathbb{N}_{0}} \frac{\left|\zeta_{j}-\zeta_{k}\right|}{\left(m+1+\zeta_{j}\right)\left(m+1+\zeta_{k}\right)} P_{m}\right) \Pi_{j}
$$

which shows that the sequence of singular values of $D_{\varepsilon, \varepsilon^{\prime}}\left(\Upsilon_{j \mapsto k}\right)$ behaves asymptotically as

$$
\mu_{m}\left[D_{\varepsilon, \varepsilon^{\prime}}\left(\Upsilon_{j \mapsto k}\right)\right]:=\frac{\left|\zeta_{j}-\zeta_{k}\right|}{\left(m+1+\zeta_{j}\right)\left(m+1+\zeta_{k}\right)} \propto(m+1)^{-2}
$$

and has multiplicity 1 . As a consequence one gets that $D_{\varepsilon, \varepsilon^{\prime}}\left(\Upsilon_{j \mapsto k}\right)$ is trace-class and in turn $D_{\varepsilon, \varepsilon^{\prime}}\left(\Upsilon_{j \mapsto k}\right) \in \mathfrak{S}^{1} \subset \mathfrak{S}_{0}^{1^{+}}$. Moreover, from the proof of [DS, Proposition 2.27] one obtains

$$
\left\|D_{\varepsilon, \varepsilon^{\prime}}\left(\Upsilon_{j \mapsto k}\right)\right\|_{1+} \leqslant\left\|Q_{B, \varepsilon}^{-1} \Upsilon_{j \mapsto k}\right\|_{1+}+\left\|\Upsilon_{j \mapsto k} Q_{B, \varepsilon}^{-1}\right\|_{1+} \leqslant 2
$$


Now, let us consider a generic element $A=\sum_{(j, k) \in \mathbb{N}_{0}^{2}} a_{j, k} \Upsilon_{j \mapsto k}$ with $\left\{a_{j, k}\right\} \in \ell^{1}\left(\mathbb{N}_{0}^{2}\right)$. The linearity of $C_{\varepsilon, \varepsilon^{\prime}}$ and the triangular inequality imply

$$
\left\|D_{\varepsilon, \varepsilon^{\prime}}(A)\right\|_{1+} \leqslant \sum_{(j, k) \in \mathbb{N}_{0}^{2}}\left|a_{j, k}\right|\left\|D_{\varepsilon, \varepsilon^{\prime}}\left(\Upsilon_{j \mapsto k}\right)\right\|_{1+} \leqslant 2\left\|\left\{a_{j, k}\right\}\right\|_{\ell^{1}}
$$

This proves that $\mathrm{D}_{\varepsilon, \varepsilon^{\prime}}: \mathscr{L}_{\mathrm{B}}^{1} \rightarrow \mathfrak{S}^{1^{+}}$is a continuous map. Since $\mathrm{D}_{\varepsilon, \varepsilon^{\prime}}(\mathrm{A}) \in \mathfrak{S}_{0}^{1^{+}}$if $\mathrm{A}$ is a finite linear combination of the operators $\Upsilon_{j \mapsto k}$, and $\mathfrak{S}_{0}^{1^{+}}$is closed in the norm \|\|$_{1+}$, one infers that $\mathrm{D}_{\varepsilon, \varepsilon^{\prime}}\left(\mathscr{L}_{\mathrm{B}}^{1}\right) \subseteq \mathfrak{S}_{0}^{1^{+}}$by continuity. The proof for the map $\mathrm{J}_{\varepsilon, \varepsilon^{\prime}, \varepsilon^{\prime \prime}}$ is similar. From the spectral resolution

$$
J_{\varepsilon, \varepsilon^{\prime}, \varepsilon^{\prime \prime}}\left(\Upsilon_{j \mapsto k}\right)=\left(\sum_{m \in \mathbb{N}_{0}} \frac{m+1+\xi_{k}-\sqrt{\left(m+1+\zeta_{j}\right)\left(m+1+\zeta_{k}\right)}}{\left(m+1+\xi_{k}\right) \sqrt{\left(m+1+\zeta_{j}\right)\left(m+1+\zeta_{k}\right)}} P_{m}\right) \Upsilon_{j \mapsto k},
$$

with $\xi_{k}:=k+\varepsilon^{\prime \prime}$, one gets

$$
\mu_{m}\left[J_{\varepsilon, \varepsilon^{\prime}, \varepsilon^{\prime \prime}}\left(\Upsilon_{j \mapsto k}\right)\right]=\frac{\left|m+1+\xi_{k}-\sqrt{\left(m+1+\zeta_{j}\right)\left(m+1+\zeta_{k}\right)}\right|}{\left(m+1+\xi_{k}\right) \sqrt{\left(m+1+\zeta_{j}\right)\left(m+1+\zeta_{k}\right)}} \propto(m+1)^{-2},
$$

which implies $J_{\mathcal{\varepsilon}, \mathcal{\varepsilon}^{\prime}, \varepsilon^{\prime \prime}}\left(\Upsilon_{j \mapsto k}\right) \in \mathfrak{S}^{1} \subset \mathfrak{S}_{0}^{1^{+}}$. Again [DS, Proposition 2.27] provides the estimate $\left\|J_{\varepsilon, \varepsilon^{\prime}, \varepsilon^{\prime \prime}}\left(\Upsilon_{j \mapsto k}\right)\right\|_{1+} \leqslant 2$. At this point, the continuity argument follows as in the previous case.

Remark A.3. From the explicit form of the singular values of $D_{\varepsilon, \varepsilon^{\prime}}\left(\Upsilon_{j \mapsto k}\right)$ provided in (A.8) one infers that

$$
\left\|D_{\varepsilon, \varepsilon^{\prime}}\left(\Upsilon_{j \mapsto k}\right)\right\|_{1} \leqslant \mathfrak{Z}(3)\left|\zeta_{j}-\zeta_{k}\right|
$$

where $\mathfrak{Z}$ denotes the Riemann zeta function. Therefore, one can use the same argument in the proof of Lemma A.6 to show that $\mathrm{D}_{\varepsilon, \varepsilon^{\prime}}: \mathscr{S}_{\mathrm{B}} \rightarrow \mathfrak{S}^{1}$ is a continuous map. A similar result also holds for $\mathrm{J}_{\varepsilon, \varepsilon^{\prime}, \varepsilon^{\prime \prime}}$.

For the next result we need to introduce the creation and annihilation operators

$$
\mathfrak{b}^{ \pm}:=-\frac{1}{\sqrt{2}}\left(\mathrm{G}_{1} \pm \mathrm{i} \mathrm{G}_{2}\right)
$$

and the number operator $N_{\mathfrak{b}}:=\mathfrak{b}^{+} \mathfrak{b}^{-}$. From the canonical commutation relation one gets $\mathfrak{b}^{-} \mathfrak{b}^{+}=N_{\mathfrak{b}}+1$. If $P_{m}$ is the dual Landau projection, then one has that $N_{\mathfrak{b}} P_{m}=$ $\mathrm{P}_{\mathrm{m}} \mathrm{N}_{\mathfrak{b}}=\mathrm{mP}_{\mathrm{m}}$.

Lemma A.4. It holds true that

$$
\mathfrak{b}^{ \pm} \mathrm{C}_{\varepsilon, \varepsilon^{\prime}}(\mathrm{A}):=\mathfrak{b}^{ \pm}\left(\mathrm{Q}_{\mathrm{B}, \varepsilon}^{-\frac{1}{2}} \mathrm{~A}-\mathrm{A} \mathrm{Q}_{\mathrm{B}, \varepsilon^{\prime}}^{-\frac{1}{2}}\right) \in \mathfrak{S}^{\mathrm{q}^{-}}, \quad \mathrm{q}>1
$$

for every $\mathrm{A} \in \mathscr{S}_{\mathrm{B}}$, independently of $\varepsilon, \varepsilon^{\prime}>-1$.

Proof. The proof follows the same strategy of Lemma A.6. Using the spectral representation of of $C_{\varepsilon, \varepsilon^{\prime}}\left(\Upsilon_{j \mapsto k}\right)$ given in [DS, Lemma 3.14] one can compute explicitly the 
singular values of $\mathfrak{b}^{ \pm} C_{\varepsilon, \varepsilon^{\prime}}\left(\Upsilon_{j \mapsto k}\right)$. One obtains that

$$
\mu_{\mathrm{m}}\left[\mathfrak{b}^{ \pm} \mathrm{C}_{\varepsilon, \varepsilon^{\prime}}\left(\Upsilon_{j \mapsto k}\right)\right]=\left(\mathrm{m}+\frac{1 \pm 1}{2}\right)^{\frac{1}{2}} \mu_{\mathrm{m}}\left[\mathrm{C}_{\varepsilon, \varepsilon^{\prime}}\left(\Upsilon_{j \mapsto k}\right)\right] \propto(\mathrm{m}+1)^{-1}
$$

where the singular values $\mu_{m}\left[C_{\varepsilon, \mathcal{E}^{\prime}}\left(\Upsilon_{j \mapsto k}\right)\right]$ are explicitly described in the proof of Lemma A.6. By using the definition of the norm \|\|$_{q^{-}}$given in Appendix A.1 one obtains that

$$
\left\|\mathfrak{b}^{ \pm} C_{\varepsilon, \mathcal{\varepsilon}^{\prime}}\left(\Upsilon_{j \mapsto k}\right)\right\|_{q^{-}} \leqslant \frac{1}{2} \mathfrak{Z}\left(2-\frac{1}{q}\right)\left|\zeta_{j}-\zeta_{k}\right|
$$

where $\mathfrak{Z}$ is the Riemann zeta function. Therefore, one has that $\mathfrak{b}^{ \pm} C_{\varepsilon, \varepsilon^{\prime}}\left(\Upsilon_{j \mapsto k}\right) \in \mathfrak{S}^{q^{-}}$ whenever $q>1$. The same argument used in the proof of Lemma A.6 provides the continuity of the maps $\mathfrak{b}^{ \pm} \mathrm{C}_{\varepsilon, \varepsilon^{\prime}}: \mathscr{S}_{\mathrm{B}} \rightarrow \mathfrak{S}^{\mathrm{q}^{-}}$and this concludes the proof.

Corollary A.5. It holds true that

$$
\mathrm{T} \mathfrak{b}^{ \pm} \mathrm{C}_{\varepsilon, \varepsilon^{\prime}}(A) \in \mathfrak{S}^{1}, \quad \mathfrak{b}^{ \pm} \mathrm{C}_{\varepsilon, \varepsilon^{\prime}}(\mathrm{A}) \mathrm{T} \in \mathfrak{S}^{1}
$$

for every $\mathrm{A} \in \mathscr{S}_{\mathrm{B}}$ and $\mathrm{T} \in \mathfrak{S}^{\mathrm{p}^{+}}$, with $\mathrm{p}>1$, independently of $\varepsilon, \varepsilon^{\prime}>-1$.

Proof. Let $p>1$ and define $q:=p /(p-1)>1$. Since $\mathfrak{S}^{p^{+}}$is the dual of $\mathfrak{S}^{q^{-}}$(see Appendix A.1) and using the result proved in Lemma A.4 one obtains $T_{\mathfrak{b}}^{ \pm} C_{\varepsilon, \varepsilon^{\prime}}(A) \in$ $\mathfrak{S}^{1}$. The second implication follows from the identity $\left(A^{*} B^{*}\right)^{*}=B A$ and the fact that $\mathfrak{S}^{\mathfrak{p}^{ \pm}}$and $\mathfrak{S}^{1}$ are self-adjoint ideals.

A.3. Quasi-symmetry of the Dirac operator and its consequences. Let us represent the Dirac operator (1.22) as the sum

$$
\mathrm{D}_{\mathrm{B}}=\mathrm{D}_{\mathrm{B},-}+\mathrm{D}_{\mathrm{B},+}
$$

of the two terms

$$
\begin{aligned}
& \mathrm{D}_{\mathrm{B},-}:=\frac{1}{\sqrt{2}}\left(\mathrm{~K}_{1} \otimes \gamma_{1}+\mathrm{K}_{2} \otimes \gamma_{2}\right), \\
& \mathrm{D}_{\mathrm{B},+}:=\frac{1}{\sqrt{2}}\left(\mathrm{G}_{1} \otimes \gamma_{3}+\mathrm{G}_{2} \otimes \gamma_{4}\right) .
\end{aligned}
$$

A simple calculation involving the commutation relations between the operators $K_{j}, G_{j}$, and the matrices $\gamma_{j}$ provides

$$
\mathrm{D}_{\mathrm{B},+} \mathrm{D}_{\mathrm{B},-}=-\mathrm{D}_{\mathrm{B},-} \mathrm{D}_{\mathrm{B},+}
$$

The latter equation immediately implies the two relations

$$
\mathrm{D}_{\mathrm{B}}^{2}=\mathrm{D}_{\mathrm{B},-}^{2}+\mathrm{D}_{\mathrm{B},+}^{2}, \quad\left[\mathrm{D}_{\mathrm{B}}^{2}, \mathrm{D}_{\mathrm{B}, \pm}\right]=0
$$

Finally, a straightforward computation provides

$$
\Gamma \mathrm{D}_{\mathrm{B}, \pm} \Gamma= \pm \mathrm{D}_{\mathrm{B}, \pm} \text {. }
$$

It is worth to point out that all the equations presented above are initially well-defined on the common core $S\left(\mathbb{R}^{2}\right) \otimes \mathbb{C}^{4}$ of $D_{B, \pm}$ and then are extended by continuity to the whole 
Hilbert space $\mathcal{H}_{4}$. The commutator of $\mathrm{D}_{\mathrm{B}}$ with elements in $\pi\left(\mathscr{S}_{\mathrm{B}}\right)$ is well-defined [DS, Proposition 3.2]. Since $\mathrm{D}_{\mathrm{B},+}$ commutes with $\pi\left(\mathscr{S}_{\mathrm{B}}\right)$ in view of [DS, Lemma 2.19] it follows that

$$
\left[\mathrm{D}_{\mathrm{B}}, \pi(\mathrm{A})\right]=\left[\mathrm{D}_{\mathrm{B},-}, \pi(\mathrm{A})\right]
$$

and in turn

$$
\Gamma\left[\mathrm{D}_{\mathrm{B}}, \pi(\mathrm{A})\right] \Gamma=-\left[\mathrm{D}_{\mathrm{B}}, \pi(\mathrm{A})\right] .
$$

The latter equation shows that $\left[D_{B}, \pi(A)\right]$ has degree 1 with respect to $\Gamma$.

In [DS, Lemma 3.10] it has been proved that $\left[\mathrm{F}_{\mathrm{B}, \varepsilon}, \pi(\mathrm{A})\right] \in \mathfrak{S}^{2^{+}}$if $\mathrm{A} \in \mathscr{S}_{\mathrm{B}}$. By replacing $\mathrm{F}_{\mathrm{B}, \varepsilon}$ with the anticommutator

$$
\left\{\Gamma, \mathrm{F}_{\mathrm{B}, \varepsilon}\right\}:=\Gamma \mathrm{F}_{\mathrm{B}, \varepsilon}+\mathrm{F}_{\mathrm{B}, \varepsilon} \Gamma=2 \Gamma \frac{\mathrm{D}_{\mathrm{B},+}}{\left|\mathrm{D}_{\mathrm{B}, \varepsilon}\right|}
$$

one obtains a stronger result.

Lemma A.6. It holds true that

$$
\left[\left\{\Gamma, F_{B, \varepsilon}\right\}, \pi(A)\right] \in \mathfrak{S}^{q^{-}}, \quad q>1
$$

for every $\mathrm{A} \in \mathscr{S}_{\mathrm{B}}$, independently of $\varepsilon>0$.

Proof. A direct computation shows that

$$
\begin{aligned}
\left\{\Gamma, \mathrm{F}_{\mathrm{B}, \varepsilon}\right\} & =2 \Gamma\left(\begin{array}{cccc}
0 & \mathfrak{b}^{+} & 0 & 0 \\
\mathfrak{b}^{-} & 0 & 0 & 0 \\
0 & 0 & 0 & -\mathfrak{b}^{-} \\
0 & 0 & -\mathfrak{b}^{+} & 0
\end{array}\right)\left|\mathrm{D}_{\mathrm{B}, \varepsilon}\right|^{-1} \\
& =2\left(\begin{array}{cccc}
0 & \mathfrak{b}^{+} \mathrm{Q}_{\mathrm{B}, \varepsilon}^{-\frac{1}{2}} & 0 & 0 \\
-\mathfrak{b}^{-} \mathrm{Q}_{\mathrm{B}, \varepsilon-1}^{-\frac{1}{2}} & 0 & 0 & 0 \\
0 & 0 & 0 & \mathfrak{b}^{-} \mathrm{Q}_{\mathrm{B}, \varepsilon}^{-\frac{1}{2}} \\
0 & 0 & \mathfrak{b}^{+} \mathrm{Q}_{\mathrm{B}, \varepsilon+1}^{-\frac{1}{2}} & 0
\end{array}\right)
\end{aligned}
$$

where the $\mathfrak{b}^{ \pm}$are the creation and annihilation operators defined by (A.9). Since the operators $\mathfrak{b}^{ \pm}$commute with $\pi(A)$ one gets that the non-zero elements of the commutator $\left[\left\{\Gamma, F_{B, \varepsilon}\right\}, \pi(A)\right]$ are of the type $\mathfrak{b}^{ \pm} C_{\varepsilon, \varepsilon}(A)$. Therefore, the result follows from Lemma A.4.

\section{APPENDiX B. THE CYCLIC COHOMOLOGY OF THE MAGNETIC ALGEBRA}

Cyclic cohomology provides a natural analog of the classical de Rham theory in the context of noncommutative $\mathrm{C}^{*}$-algebras. A complete description of this theory is presented in [Con, Chapter 3] and [GVF, Chapters $8 \& 10]$. In this section, we will review only the most basic aspects of the theory. 
To describe the cyclic cohomology of the magnetic algebra we start by considering the set of $(n+1)$-multilinear functionals $\varphi$ defined on $\mathscr{S}_{\mathrm{B}}$, satisfying the cyclic condition

$$
\varphi\left(A_{1}, \ldots, A_{n}, A_{0}\right)=(-1)^{n} \varphi\left(A_{0}, A_{1}, \ldots, A_{n}\right), \quad A_{i} \in \mathscr{S}_{B},
$$

Let $\mathrm{C}_{\lambda}^{n}\left(\mathscr{S}_{\mathrm{B}}\right)$, with $\mathrm{n} \in \mathbb{N}_{0}$, be the linear space of cyclic densely defined $(\mathrm{n}+1)$-linear functionals. The elements of $\mathrm{C}_{\lambda}^{n}\left(\mathscr{S}_{\mathrm{B}}\right)$ are called cyclic $n$-cochains. On the family of sets $\mathrm{C}_{\lambda}^{\mathrm{n}}\left(\mathscr{S}_{\mathrm{B}}\right)$ acts the Hochschild coboundary map b : $\mathrm{C}_{\lambda}^{\mathrm{n}}\left(\mathscr{S}_{\mathrm{B}}\right) \rightarrow \mathrm{C}_{\lambda}^{\mathrm{n}+1}\left(\mathscr{S}_{\mathrm{B}}\right)$ given by

$$
\begin{aligned}
(b \varphi)\left(A_{0}, \ldots, A_{n+1}\right)= & \sum_{j=0}^{n}(-1)^{n} \varphi\left(A_{0}, \ldots, A_{j} A_{j+1}, \ldots, A_{n+1}\right) \\
& +(-1)^{n+1} \varphi\left(A_{n+1} A_{0}, \ldots, A_{n+1}\right) .
\end{aligned}
$$

From the definition, one gets $b^{2}=0$. An element $\varphi \in C_{\lambda}^{n}\left(\mathscr{S}_{\mathrm{B}}\right)$ is called cyclic $\mathrm{n}$ cocycle, if and only if, $\mathrm{b} \varphi=0$. Elements of the form $\mathrm{b} \varphi \in \mathrm{C}_{\lambda}^{\mathrm{n}}\left(\mathscr{S}_{\mathrm{B}}\right)$ are called cyclic $\mathrm{n}$-coboundaries. The cyclic cohomology of $\mathscr{S}_{\mathrm{B}}$ is the cohomology of the cyclic complex $\left(\mathrm{C}_{\lambda}^{\bullet}\left(\mathscr{S}_{\mathrm{B}}\right), \mathrm{b}\right)$, and it is denoted by $\mathrm{HC} \bullet\left(\mathscr{S}_{\mathrm{B}}\right)$. More precisely one has that

$$
\mathrm{HC}^{\mathrm{n}}\left(\mathscr{S}_{\mathrm{B}}\right)=\frac{\operatorname{Ker}\left(\mathrm{b}: \mathrm{C}_{\lambda}^{\mathrm{n}}\left(\mathscr{S}_{\mathrm{B}}\right) \rightarrow \mathrm{C}_{\lambda}^{\mathrm{n}+1}\left(\mathscr{S}_{\mathrm{B}}\right)\right)}{\operatorname{Ran}\left(\mathrm{b}: \mathrm{C}_{\lambda}^{\mathrm{n}-1}\left(\mathscr{S}_{\mathrm{B}}\right) \rightarrow \mathrm{C}_{\lambda}^{\mathrm{n}}\left(\mathscr{S}_{\mathrm{B}}\right)\right)}, \quad \mathrm{n} \in \mathbb{N}_{\mathrm{o}} .
$$

Any element of $\mathrm{HC}^{n}\left(\mathscr{S}_{\mathrm{B}}\right)$ is an equivalence class of cyclic $n$-cocycles modulo cyclic n-coboundaries.

Let us recall that there exist the periodicity operator $\mathrm{S}$ which provides group homomorphisms $\mathrm{S}: \mathrm{HC}^{\mathrm{n}}\left(\mathscr{S}_{\mathrm{B}}\right) \rightarrow \mathrm{HC}^{\mathrm{n}+2}\left(\mathscr{S}_{\mathrm{B}}\right)$ [GVF, Section 10.1]. Using this operator, one obtains two groups as the inductive limits

$$
\mathrm{HC}^{\text {even }}\left(\mathscr{S}_{\mathrm{B}}\right):=\underset{\lim }{\longrightarrow} \mathrm{HC}^{2 \mathrm{n}}\left(\mathscr{S}_{\mathrm{B}}\right), \quad \mathrm{HC}^{\text {odd }}\left(\mathscr{S}_{\mathrm{B}}\right):=\underline{\lim } \mathrm{HC}^{2 \mathrm{n}+1}\left(\mathscr{S}_{\mathrm{B}}\right) .
$$

which define the periodic cyclic cohomology of $\mathscr{S}_{\mathrm{B}}$. The next result is essentially proved in $[\mathrm{ENN}]$.

\section{Lemma B.1. It holds true that}

$$
\mathrm{HC}^{\text {even }}\left(\mathscr{S}_{\mathrm{B}}\right)=\mathbb{Z}\left[f_{\mathrm{B}}\right], \quad \operatorname{HC} C^{\text {odd }}\left(\mathscr{S}_{\mathrm{B}}\right)=0 .
$$

Proof. In [ENN, Theorem 2] it is proved that $\mathrm{HC}^{\star}\left(\mathscr{K}^{\infty}\right)=\mathrm{HC}^{\star}(\mathbb{C})$ where $\star$ stays for even or odd and $\mathscr{K}^{\infty}$ denotes the $*$-algebra of those Hilbert-Schmidt operators on $\mathrm{L}^{2}(\mathbb{R})$ whose integral kernels belong to $S\left(\mathbb{R}^{2}\right)$. By adapting [Fol1, Theorem 1.30] one obtains that the "Weyl transform" $\rho$ provides a $*$-isomorphism $\mathscr{S}_{\mathrm{B}} \simeq \mathscr{K}^{\infty}$. As a consequence one has that the periodic cyclic cohomology of $\mathscr{S}_{\mathrm{B}}$ coincides with that of $\mathbb{C}$ which is known to be $\mathrm{HC}^{\text {even }}(\mathbb{C}) \simeq \mathbb{Z}$ and $\mathrm{HC}^{\text {odd }}(\mathbb{C})=0$. To conclude the proof it is enough to observe that a cyclic 0 -cocycle is clearly the same thing as a trace and $\mathscr{S}_{\mathrm{B}}$ is endowed with the (faithful) trace $f_{\mathrm{B}}$.

There are, in principle, two canonical pairings between periodic cyclic cohomology and K-theory [Con, Section 3.III]. In the specific case of the $*$-algebra $\mathscr{S}_{\mathrm{B}}$ the only relevant 
pairing is

$$
\langle,\rangle: \operatorname{HC}^{\text {even }}\left(\mathscr{S}_{\mathrm{B}}\right) \times \mathrm{K}_{0}\left(\mathscr{S}_{\mathrm{B}}\right) \rightarrow \mathbb{C}
$$

implemented by

$$
\langle[\varphi],[\mathrm{P}]\rangle:=\frac{1}{\mathrm{~m} !}\left(\varphi \sharp \operatorname{Tr}_{\mathbb{C}^{\mathrm{N}}}\right)(\mathrm{P}, \ldots, \mathrm{P}),
$$

where $\varphi \in \mathrm{C}_{\lambda}^{2 m}\left(\mathscr{S}_{\mathrm{B}}\right)$ is a representative of $[\varphi] \in \operatorname{HC}^{\text {even }}\left(\mathscr{S}_{\mathrm{B}}\right)$ and the projection $\mathrm{P} \in$ $\mathscr{S}_{\mathrm{B}} \otimes \operatorname{Mat}_{\mathrm{N}}(\mathbb{C})$ is a representative of $[\mathrm{P}] \in \mathrm{K}_{0}\left(\mathscr{S}_{\mathrm{B}}\right)$. The odd pairing is trivial in view of the fact that $\operatorname{HC}$ odd $\left(\mathscr{S}_{\mathrm{B}}\right)=0=\mathrm{K}_{1}\left(\mathscr{S}_{\mathrm{B}}\right)$.

\section{REFERENCES}

[AM] Alberti, P. M.; Matthes, R., Connes' Trace Formula and Dirac Realization of Maxwell and Yang-Mills Action. Noncommutative Geometry and the Standard Model of Elementary Particle Physics, Lecture Notes in Physics 596 (F. Scheck, W. Werner and H. Upmeier eds.). Springer, Berlin, 2002, pp. 40-74

[AMSZ] Azamov, N.; McDonald, E.; Sukochev, F.; Zanin, D.: A Dixmier trace formula for the density of states. Commun. Math. Phys. 377, 2597-2628 (2020)

[BES] Bellissard, J.; van Elst, A.; Schulz-Baldes, H.: The Non-Commutative Geometry of the Quantum Hall Effect. J. Math. Phys. 35, 5373-5451 (1994)

[Bel1] Bellissard, J.: K-theory of C*-algebras in solid state physics. Statistical Mechanics and Field Theory: Mathematical Aspects. Lecture Notes in Physics 257 (edit. by Dorlas, T.; Hugenholtz, M.; Winnink, M.). Springer-Verlag, Berlin, pp. 99-156, 1986

[Bel2] Bellissard, J.: Gap labelling theorems for Schrödinger operators. From Number Theory to Physics. Springer, Berlin, pp. 538-630, 1993

[Bou] Bourne, C.: Private communication. (2020)

[BS] Birman, M.; Solomjak, M. Z.: Spectral Theory of Self-Adjoint Operators in Hilbert Space. D. Reidel Publishing Co., Dordrecht, 1987

[CM] Connes, A., Moscovici, H., The local index formula in noncommutative geometry. Geom. Func. Anal. 5, 174-243 (1995)

[Con] Connes, A.: Noncommutative Geometry. Academic Press, 1994

[DGM] De Nittis, G.; Gomi, K.; Moscolari, M.: The geometry of (non-abelian) landau levels. J. Geom. Phys. 152, 103649 (2020)

[DS] De Nittis, G.; Sandoval, M.: The noncommutative geometry of the Landau Hamiltonian: Metric aspects. SIGMA 16, 146 (2020)

[ENN] Elliot, G. A.; Natsume, T.; Nest, R.: Cyclic cohomology for one-parameter smooth crossed products. Acta Math. 160, 285-305 (1988)

[Fol1] Folland, G. B.: Harmonic Analysis in Phase Space. Princeton University Press, Princeton, 1989

[Gree] Greenleaf, F. P.: Invariant Means on Topological Groups And Their Applications. Van Nostrand Reinhold Co., New York, 1969

[GVF] Gracia-Bondia, J. M., Varilly, J. C., Figueroa, H.: Elements of Noncommutative Geometry. Birkhäuser, Boston, 2001

[LLW] Loring T. A., Lu, J.; Watson, A. B.: Locality of the windowed local density of states. preprint arXiv:2101.00272, (2021)

[LSB1] Loring T. A., Schulz-Baldes, H.: Finite volume calculation of $K$-theory invariants. New York J. Math. 22, 1111-1140 (2017)

[LSB2] Loring T. A., Schulz-Baldes, H.: Spectral Flow Argument Localizing an Odd Index Pairing. Canad. Math. Bull. 62, 373-381 (2019) 
[LSB3] Loring T. A., Schulz-Baldes, H.: The spectral localizer for even index pairings. J. Noncommut. Geom. (electronically published) (2020)

[LSZ] Lord, S., Sukochev, F., Zanin, D., Singular Traces: Theory and Applications. De Gruyter, Berlin, 2012

[Pie] Pietsch, A.: Weyl Numbers and Eigenvalues of Operators in Banach Spaces. Math. Ann. 247, 149-168 (1980)

[Sim1] Simon, B.: Analysis With Weak Trace Ideals and the Number of Bound States of Schrödinger Operators. Trans. Amer. Math. Soc. 224, 367-380 (1976)

[Sim2] Simon, B.: Trace Ideals and Their Applications. AMS, Providence, 2005

[Ves] Veselić, I.: Existence and Regularity Properties of the Integrated Density of States of Random Schrödinger Operators. Springer, Berlin-Heidelberg, 2008

[Xia] Xia, J.: Geometric Invariants of the Quantum Hall Effect. Commun. Math. Phys. 119, 29-50 (1988)

(G. De Nittis) Facultad de Matemáticas \& Instituto de Física, Pontificia Universidad Católica de Chile, Santiago, Chile.

Email address: gidenittis@mat.uc.cl

(M. Sandoval) Facultad de Matemáticas, Pontificia Universidad Católica de Chile, SAntiago, Chile.

Email address: msandova@protonmail.com 